OBETS. Revista de Ciencias Sociales

Vol. 16, no 2, 2021, pp. 481-506

ISSN: 1989-1385

https://doi.org/10.14198/OBETS2021.16.2.16

\title{
REVISIÓN SISTEMÁTICA DE LA EVALUACIÓN DE LA MENTORÍA SOCIAL DIRIGIDA A JÓVENES VULNERABLES
}

\author{
SYSTEMATIC REVIEW OF EVALUATIONS OF SOCIAL MENTORING \\ PROGRAMMES FOR VULNERABLE YOUTH
}

\author{
Anna Sánchez-Aragón \\ Universitat Rovira i Virgili, España \\ annamaria.sanchez@urv.cat \\ https://orcid.org/0000-0002-9165-651X \\ Angel Belzunegui-Eraso \\ Universitat Rovira i Virgili, España \\ angel.belzunegui@urv.cat \\ https://orcid.org/0000-0002-6355-1593
}

Òscar Prieto-Flores

Universitat de Girona, España

oscar.prieto@udg.edu

https://orcid.org/0000-0003-4162-6109

\begin{abstract}
Cómo citar / Citation: Sánchez-Aragón, A., Belzunegui-Eraso, A. y Prieto-Flores, Ó. (2021) "Revisión sistemática de la evaluación de la mentoría social dirigida a jóvenes vulnerables". OBETS. Revista de Ciencias Sociales, 16(2): 481-506. https://doi.org/10.14198/ OBETS2021.16.2.16
\end{abstract}

\section{(C) 2021 Anna Sánchez-Aragón, Angel Belzunegui-Eraso, Òscar Prieto-Flores}

Este es un artículo de acceso abierto distribuido bajo los términos de la licencia de uso y distribución Creative Commons Reconocimiento 4.0 Internacional (CC BY 4.0) https://creativecommons.org/licenses/by/4.0/deed.es

Recibido: 03/02/21. Aceptado: 14/04/21

\section{Resumen}

Este artículo ofrece una revisión sistemática de las distintas metodologías, técnicas y estrategias en la investigación evaluativa de la mentoría social dirigida a jóvenes vulnerables. Se analizan treinta y cinco evaluaciones de programas durante la década 2010-2020. Los resultados obtenidos muestran el uso mayoritario de una metodología experimental con ensayos controlados y aleatorizados. Este tratamiento metodológico se está viendo progresivamente complementado por la incorporación de investigaciones cualitativas centradas en entender la experiencia de los jóvenes dentro de ella. En base a estos resultados, se plantea la pluralidad de métodos como alternativa en el campo de la mentoría y se destaca la necesidad de contar con estudios que informen sobre la calidad del proceso.

Palabras clave: Mentoría social, jóvenes vulnerables, evaluación, RCT, métodos mixtos.

\begin{abstract}
In this paper we conduct a systematic review of the scientific literature on the methodologies, techniques and strategies employed in evaluative research into social mentoring programmes for vulnerable youth. To do so, we analyse thirtyfive evaluations of programmes published between 2010 and 2020. Our results show that most evaluations employ a methodology whose experimental design is in the form of controlled randomised trials. This methodological treatment is now being complemented by new qualitative research that reveals an interest in understanding the experience of young people within it. On the basis of these results, we propose the plurality of methods as an alternative in the field of mentoring and stress the need for studies that will provide information about process quality.
\end{abstract}

Keywords: Social mentoring, vulnerable youth, evaluation, RCT, mixed methods. 


\section{Extended abstract}

\section{Background}

The expansion of social mentoring programmes has outpaced evidence showing their effectiveness. Although most of these initiatives continue to be concentrated in the United States, with more than 2.5 million volunteers involved every year (Raposa, Dietz and Rhodes, 2017), countries like Israel, Canada, the United Kingdom, Australia, New Zealand and, more recently, Continental Europe are beginning to incorporate mentoring projects that seek the social inclusion and well-being of the most vulnerable groups into their political agendas (Preston, Prieto-Flores and Rhodes, 2019). This growth suggests that there is now more knowledge with regards to which mentoring models work, under what conditions and for whom. However, the results of existing meta-analyses in the field indicate a modest impact of the programmes (DuBois et al., 2002, 2011; Raposa et al., 2019). Some authors, such as Brumovská and Seidlová Málková (2020), attribute this lack of knowledge to the hegemonic use of quantitative methodologies. Studies that apply an experimental design based on tests with randomized control are usually the most common in evaluating the effects of social mentoring, although whether or not this technique is the most suitable has not been properly debated (Brady and O'Regan, 2009).

This article carries out a systematic review of the literature focused on exploring the methodological plurality present in scientific evaluations that measure the impact of social mentoring programmes aimed at vulnerable youth. Specifically, it explores the diversity of research published in the last ten years and shows the different evaluative approaches, as well as the tools and experiences aimed at determining the quality of the programmes. Two main approaches stand out: the quantitative approach, aimed at measuring the impact through statistical techniques and randomized designs; and the qualitative approach, based on interviews, focus groups and observation, which attempts to understand the nature of the dyadic relationship from individual experiences.

\section{Methodology}

The quantitative systematic literature review was conducted using the procedure outlined by Pickering and Byrne (2013), a methodology that not only describes the contribution of the relevant literature, but also detects knowledge gaps that remain in this area. The selection of these studies corresponds to the last ten years - from 2010 to today-a period in which the scientific literature has grown exponentially. To find the most relevant articles that referred to this topic, searches were carried out in Scopus, Google Scholar and Web of Science (WoS) with the terms "Mentoring" or "Youth-Mentoring" separately or combining them with other words such as "Evaluation", "Assessment" or "Longitudinal research".

The criteria used for the selection of the scientific articles and books focused on: a) those studies available in full text, published since 2010; b) the characteristics of the type of social mentoring programmes evaluated -one-on- one, group, peer or youth-initiated mentoring; c) the target population -adolescents and young people aged 26 or younger at risk of social exclusion; and d) the methods used to assess the quality of the mentoring relationship, whether based solely on randomized controlled trials or on other quantitative, qualitative or mixed methods. In total, thirtyfive evaluations of mentoring programmes were analysed. Some of these were meta-analyses of a further two hundred and three evaluations. The analytical corpus on which this article is based therefore comprises two hundred and thirtyeight evaluations.

\section{Main findings}

Results from the study showed that $93.6 \%$ of the research analysed in this literature review, taking the meta-analyses into account as well, are based on quantitative methods. Few studies used mixed methods, and even fewer only qualitative methods. In fact, half of the selected articles used experimental methods in the form of randomized controlled trials. This methodological tool can determine the mechanisms that in a mentoring programme connect cause and effect if the study subjects follow the programme to which they have been assigned randomly. In this case, the pretest-posttest design with an equivalent control group is common, which allows the results to be defined and quantified before and after receiving mentoring. One of the studies that allowed the longest time to elapse between the pre and posttest (five years) was the exploration carried out by Rodríguez-Planas (2012) of the project Quantum Opportunity, which is dedicated to promoting access to higher education for young people at risk of social exclusion.

The methodological homogeneity — with the predominance of quantitative methods and, more specifically, experimental designs based on randomized controlled trials- has been addressed with the incorporation of new qualitative research. In contrast to experimental and quasi-experimental methods, qualitative studies aim to understand the unfolding of relationships and the meaning they acquire over time. Some of the empirical studies reviewed based on interviews were able to document not only the end of the mentoring relationship, its causes and consequences (Spencer et al., 2017, 2020), but also how social support can play a key role in the life of mentees, helping them, for example, to cope with specific situations or stressors, such as perceived discrimination in the immigrant population (Griffith, Hurd and Hussain, 2019); or to access new networks of social capital that can reduce the feeling of marginality of other vulnerable groups, such as people with disabilities (Stewart, Barnfather, MagillEvans, Ray and Letourneau, 2011) or disadvantaged and low income urban sectors (Sánchez et al., 2011, 2018).

The most frequent method of qualitative research is the interview, although many researchers prefer to integrate it in a multiple methods design; for example Singh and Tregale (2015), who combine the use of focus groups and semistructured interviews. The use of more than one research method offers the potential for a more complex understanding of the mentoring relationship, although the combination of qualitative and quantitative strategies is rare in this field. A 
recent example is the study by Dutton, Deane and Bullen (2018) who, as well as combining discussion groups with the staff of the mentoring programme and questionnaires for the participants, their research design attached importance to other tools such as the use of portfolios, which places emphasis on monitoring the relationship. Another method used in mentoring research is the case study which, among others, uses observation techniques and discourse analysis in order to gain a better understanding of the participants' experience (Stumbo et al., 2010).

The empirical evidence shows that many and very varied relationships are forged within a particular programme, in which not all the participants manage to establish a strong affective bond with their mentor. Hence the importance of new approaches that try to understand how and why mentoring experiences differ from one another, with some yielding negative and others positive results. Ethnography as an approach allows an external observer the possibility of participating as a silent witness to the encounters, observing many different situations that would otherwise be impossible. One recent study that clearly reflects the contributions of ethnography in the field is that of Barrett Cox (2017), which highlights the way in which specific organizational structures can enable youth at risk of social exclusion to benefit from social capital networks that help them gain access to university.

\section{Conclusions}

Although there is currently a considerable volume of robust evidence that attempts to explain social mentoring relationships, research is dominated by experimental design based on randomized controlled trials that, while they establish cause-effect relationships, can miss new knowledge about the process and nature of the bonds forged between mentor and mentee that can be gained through other methods. In accordance with this and in contrast to the hegemonic use of randomized controlled trials, this study proposes methodological plurality as an encouraging alternative in the field of social mentoring, although other studies are needed to identify the appropriate way to combine and integrate quantitative and qualitative methods. The future challenge for mentoring research consists in developing mixed methods designs that not only provide a quantitative and generalizable perspective, allusive to patterns that provide evidence for decision-making, but that can also include a more interpretive dimension that, on considering the subjective perspective, places attention on what the young people experience in their encounters. This methodological proposal allows us to understand the impact of social mentoring through an approach focused on processes but also on results.

\section{INTRODUCCIÓN}

Aunque existe una amplia variación entre los servicios prestados por los programas de mentoría, en el contexto de este artículo, la mentoría se define como un método de intervención social que fomenta la relación entre personas que de manera voluntaria se prestan para ofrecer ayuda individual a otra — protégéque se encuentra en riesgo de exclusión. Como esta, en la literatura científica se recogen muchas definiciones de mentoría, todas ellas útiles y con al menos dos elementos en común: el primero, que el mentor tiene mayor experiencia que el mentorado; y el segundo, que el mentor ofrece una guía o apoyo que puede facilitar el desarrollo y las competencias personales del que lo recibe. Otras definiciones también reconocen que suele existir un vínculo emocional entre mentor y mentorado, basado en la cercanía y la confianza mutua (DuBois y Karcher, 2005).

La expansión de los programas de mentoría social ha ido por delante de la evidencia de su eficacia. Si bien Estados Unidos sigue aglutinando la mayoría de estas iniciativas, con más de 2,5 millones de voluntarios involucrados cada año (Raposa, Dietz y Rhodes,

2017); países como Israel, Canadá, Reino Unido, Australia, Nueva Zelanda y, más recientemente, Europa Continental están empezando a incorporar en sus agendas políticas proyectos de mentoría que buscan la inclusión social y el bienestar de los colectivos más vulnerables (Preston, Prieto-Flores y Rhodes, 2019). Este crecimiento hace suponer que ahora se tienen mayores conocimientos con respecto a qué modelos de mentoría funcionan, bajo qué condiciones lo hacen y para quién; sin embargo, los académicos sostienen que existen ciertas dificultades para entender por qué determinados programas pueden llegar a tener resultados negativos e incluso perjudiciales.

La voluntad de dar respuesta a estas lagunas de conocimiento ha hecho que los investigadores lleven a cabo nuevos estudios orientados a comprender mejor la naturaleza y el desarrollo de las relaciones, cuyas implicaciones para la eficacia de la mentoría pueden ayudar a la creación de proyectos más satisfactorios. Los resultados de los metaanálisis existentes en el campo demuestran que la mentoría suele mejorar el desarrollo emocional, académico y conductual de las personas en riesgo de exclusión social (Prieto-Flores y Feu, 2018), pero denotan un impacto modesto de los programas 
(DuBois et al., 2002, 2011). Las investigaciones sobre los procesos relacionales, aunque son cada vez más frecuentes y han contribuido enormemente a nuestra comprensión de las prácticas asociadas con actuaciones más eficaces, aún no han conseguido mejorar el tamaño de sus efectos (Raposa et al., 2019).

Algunos autores, como Brumovská y Seidlová Málková, (2020) atribuyen esta falta de conocimiento al uso hegemónico de metodologías cuantitativas. Los estudios que aplican un diseño experimental basado en pruebas con control aleatorizado suelen ser los más comunes en la evaluación de los efectos de la mentoría social, pese a que no se ha debatido a fondo que esta sea la técnica que mejor se ajuste (Brady y O'Regan, 2009). De hecho, estudios como el de Pryce, Deane, Barry y Keller (2020) sugieren que el empleo en exceso de cuestionarios de autoinforme dificulta llevar a cabo evaluaciones más precisas, puesto que los participantes aplican sus propios significados e interpretaciones idiográficas.

Desde la investigación de Tierney, Grossman y Resch a mediados de la década de los noventa acerca del programa Big Brothers Big Sisters of America —en adelante BBBSA - en los Estados Unidos, han sido muchos los estudios que han prestado atención al uso de metodologías de diseño experimental y han intentado replicar este tipo de evaluación. Por ejemplo, la investigación realizada por Dolan, Brady, O'Regan, Canavan, Russell y Forkan (2011) sobre el programa Foróige, la versión irlandesa del BBBSA. Esta tendencia sitúa los ensayos controlados y aleatorizados como el método por antonomasia para evaluar la mentoría social, pese a las limitaciones que algunos autores, como Deutsch y Spencer (2009), relacionan con esta técnica. Según éstas, una desventaja de los estudios de diseño experimental basados en el impacto es que puede pasar por alto la naturaleza fluida y cambiante de los factores que hacen que una relación de mentoría se malogre o tenga éxito. Esta opción metodológica, de acuerdo con las autoras, no entiende el trascurso de las relaciones $y$, en su defecto, mediante indicadores de entrada y salida, proporciona una instantánea de los efectos de la mentoría en un momento y tiempo concretos.

Otra de las limitaciones que se observan de los ensayos controlados aleatoriamente es que sólo explora las diferencias que pueda haber entre dos grupos —uno experimental y otro de control- en un periodo de tiempo que rara vez supera los quince meses (Prieto-Flores y Feu, 2018). Los estudios que aportan evidencias sobre los efectos de la mentoría a largo plazo son escasos. El coste elevado y la gran cantidad de tiempo que requieren hacen que pocos académicos se animen a emprender estudios de estas características, pese a las importantes implicaciones de los hallazgos, que podrían aportar nuevas evidencias acerca de la persistencia de los efectos de los programas. Los estudios longitudinales son imprescindibles para averiguar si la mentoría produce un impacto a largo plazo y si tiene consecuencias estructurales en la vida de los participantes. En el contexto norteamericano, el estudio de los mentores naturales que llevaron a cabo DuBois y Silverthorn (2005), a partir de la base de datos pública sobre salud adolescente (Add Health), observó que los efectos continuaban más allá de la mentoría. Los autores concluyeron que los jóvenes adultos que habían tenido una relación con un mentor durante la adolescencia mostraban una mejor salud mental —autoestima y satisfacción con la vida- y mayores resultados asociados con la trayectoria académica y laboral. Sin embargo, la carencia de estudios longitudinales que lleven a cabo un seguimiento de la transición a la vida adulta de los participantes impide dar respuesta a cómo y de qué manera los efectos de los programas persisten con el paso del tiempo.

Raposa, Ben-Eliyahu, Olsho y Rhodes (2018) llaman también la atención sobre los inconvenientes del uso de variables dicotómicas en evaluaciones cuantitativas de algún constructo en particular de las relaciones de mentoría. Según los autores, un problema específico es que implica respuestas forzadas. El sesgo en los instrumentos de medición es una preocupación generalizada que ha hecho inclinar la balanza hacia el uso de escalas tipo Likert, o politómicas, puesto que se asume que un mayor número de opciones de respuesta permitirá obtener mayor información del individuo. Desde su aparición, cada vez son más los investigadores que cuentan con este método para la construcción de instrumentos de evaluación (Harris y Nakkula, 2018; Rhodes, Schwartz, Willis y $\mathrm{Mu}, 2017$; Zand et al., 2009; Rhodes, Reddy, Roffman, y Grossman, 2005). No obstante, un riesgo evidente de las técnicas cuantitativas es que impiden que surjan categorías emergentes de análisis —no previstas en la formulación original por el investigador-que pueden aportar información muy valiosa acerca de la naturaleza de las relaciones. 
En este artículo se aborda una revisión sistemática de la literatura centrada en explorar la pluralidad metodológica presente en las evaluaciones científicas que miden el impacto de los programas de mentoría social dirigida a jóvenes vulnerables. En concreto, la novedad que presenta esta revisión es la exploración de la diversidad de investigaciones publicadas en los últimos diez años, centrándonos en las evaluaciones realizadas de las experiencias de mentoría. A diferencia de otras revisiones sistemáticas, en esta que presentamos aquí el $65,7 \%$ de la literatura ha sido publicada en los últimos cinco años, periodo que coincide con el aumento de los programas de mentoría social en Europa (Preston, Prieto-Flores y Rhodes, 2019). Los metaanálisis y las revisiones de literatura existentes hasta el momento recogen en su mayoría estudios de programas desarrollados con anterioridad al 2010, por lo que se considera necesario revisar la literatura más reciente con la idea de actualizar el conocimiento que se tiene. Por otra parte, la mayoría de estos estudios se han realizado en lengua inglesa, de modo que la publicación de este artículo en castellano supone también una novedad en sí misma. En esta revisión de la literatura se muestran los diferentes enfoques evaluativos que se están llevando a cabo, así como las herramientas y experiencias encaminadas a determinar la calidad de los programas. Se destacan dos enfoques principales: la evaluación de enfoque cuantitativo, volcada en medir el impacto a través de técnicas estadísticas y de diseños aleatorizados, y la evaluación de enfoque cualitativo, basada en entrevistas, grupos focales y observación, que trata de entender la naturaleza de la relación diádica a partir de las experiencias individuales. Por último, se concluye con una serie de reflexiones que permiten diagnosticar los retos y limitaciones que definen los actuales métodos de evaluación, así como posibles líneas de acción para el avance de la mentoría social en este campo.

\section{MÉTODO}

Para desarrollar esta revisión sistemática de literatura se ha utilizado la Systematic Quantitative Literature Review - en adelante SQLR_- una metodología que no sólo permite describir la contribución de la literatura relevante, sino también detectar los vacíos teóricos o huecos de conocimiento que quedan por analizar. Su método sencillo de categorización aporta información organizada sobre en qué lugares existen datos, en qué países se están desarrollando los estudios empíricos, en qué años o qué metodologías se utilizan más en las investigaciones sobre un tema específico (Pickering y Byrne, 2013).

La SQLR es un sistema que comprende una serie de pasos interrelacionados (Pickering y Byrne, 2013). En primer lugar, a partir de las preguntas de investigación, y teniendo en cuenta las posibles variaciones ortográficas y sinónimas, se definieron las palabras clave (keywords) que más se ajustaban a la temática del estudio. Estos términos de búsqueda permitieron reunir artículos científicos sobre un mismo tema en índices y bases de datos bibliográficas. Una vez concluida esta tarea, se realizó la lectura de los documentos para determinar su pertinencia y desechar los que no se ajustaban al objeto de estudio. El siguiente paso consistió en crear una base de datos, mediante una hoja de cálculo Excel, que permitió compilar y gestionar de forma homogénea la información de todos los artículos seleccionados. En esta base de datos se introdujeron las referencias bibliográficas por filas y se categorizaron en columnas de acuerdo a las variables relevantes para el análisis, como el método de investigación utilizado, las características de la muestra o la ubicación geográfica del estudio empírico. A continuación, con el fin de probar el formato de extracción de datos, se llevó a cabo un ensayo previo, consistente en introducir y analizar el 10\% de todos los artículos encontrados. Este ejercicio permitió revisar las categorías definidas por si fuera preciso una modificación en la base de datos. Una vez completado este proceso, se introdujeron todos los artículos y se analizaron los datos resultantes mediante resúmenes cuantitativos y/o gráficos y la selección y categorización de la información.

\subsection{Base de datos}

En los últimos años, debido al creciente número de programas de mentoría, han emergido cuantiosas investigaciones científicas que se han centrado en examinar los efectos de este método de intervención en la inclusión social de las personas en riesgo. El presente artículo explora los enfoques evaluativos, así como las técnicas y recursos metodológicos, que existen para medir la calidad de las relaciones.

La selección de estos estudios científicos corresponde a los últimos diez años —desde 2010 hasta 
la actualidad-, en los que la bibliografía científica ha crecido exponencialmente. Para alcanzar un análisis exhaustivo, y con el fin de abordar estudios realizados a escala global, no se han aplicado limitaciones geográficas, lo que ha permitido observar diferentes fórmulas y estrategias de evaluación de programas en un amplio espectro de estudio de casos. Para ajustar la selección de artículos referentes a este tema, se llevaron a cabo búsquedas en Scopus, Google Scholar y Web of Science (WoS) con el término «Mentoring» por separado o combinándolo con otras palabras que hicieran hincapié en la relación de mentoría y su calidad de acuerdo con distintos factores, como la duración o la frecuencia de los encuentros; aludieran a los adolescentes y jóvenes como destinatarios de este método de intervención, por ejemplo «Youth-Mentoring»; y tuvieran que ver con la evaluación de los programas de mentoría, también a largo plazo, como fueron «Evaluation», «Assessment» o «Longitudinal research». En base a las palabras clave identificadas, se definió una secuencia de búsqueda más compleja utilizando los operadores booleanos AND/ OR y el carácter comodín ${ }^{*}$ ) para encontrar cualquier palabra en su forma singular o plural. Por ejemplo, una secuencia de búsqueda resultante fue: (YouthMentoring AND assesment) OR (Youth-Mentoring AND evaluation). Esta cadena de consulta se adaptó a cada una de las palabras clave, lo que resultó en una configuración de búsqueda muy exhaustiva por cada base de datos bibliográfica utilizada.

\subsection{Criterios de selección, extracción de datos y análisis}

La búsqueda se limitó a artículos científicos publicados en las bases de datos habituales, lo que excluyó a la literatura gris de la síntesis de investigaciones. Las fuentes de documentación gris no suelen estar revisadas por pares ni ajustarse necesariamente a las normas de control bibliográfico, por lo que pueden plantear problemas de validez. Los criterios empleados para la selección de los artículos científicos y libros se centraron en: (a) aquellos estudios disponibles en texto completo, publicados a partir del año 2010; (b) las características de los tipos de programa de mentoría social evaluados - mentoría uno a uno, grupal, entre pares o youth-initiated mentoring-; (c) la población destinataria -adolescentes y jóvenes menores o iguales a 26 años en riesgo de exclusión social-; y (d) los métodos utilizados para evaluar la calidad de la relación de mentoría, ya estuvieran basados únicamente en ensayos controlados y aleatorizados o en otras técnicas cuantitativas, cualitativas o mixtas. Por otro lado, los criterios de exclusión fueron: (a) los artículos que no pertenecían específicamente al área de interés de la mentoría social, cuyos objetivos diferían al análisis de la evaluación de las relaciones; (b) los que tuvieran metodologías incompletas o poco explícitas; y (c) los escritos en idiomas diferentes al inglés.

De acuerdo a los criterios de selección, se diseñó un formulario de extracción de datos como instrumento para revisar con toda precisión la información de los artículos. Cada uno fue codificado en múltiples características, que podían dividirse en cinco categorías distintas: (1) características generales del estudio autor, año de publicación y país-; (2) características de la población —edad, sexo, raza/etnia y nivel de riesgo-; (3) tipo de mentoría - uno a uno, grupal, entre pares o youth-initiated mentoring-; (4) características metodológicas — tamaño de muestra y diseño—, y (5) resultados. En la búsqueda inicial se identificaron 268 artículos. Después de eliminar los estudios publicados antes del $2010(n=113)$ y aquellos cuyo objeto de estudio eran personas mayores de 26 años $(n=16)$, quedaron 139. Tras descartar los artículos con objetivos distintos a la evaluación de las relaciones de mentoría ( $n=77)$ y duplicados $(n=6)$, el número de artículos se redujo a 56, de los cuales 21 fueron eliminados por presentar una metodología inespecífica. En total se analizaron treinta y cinco evaluaciones de programas de mentoría, el 65,7\% publicadas en los últimos cinco años. Algunos de estos trabajos son metaanálisis que recogen otras 203 evaluaciones. Esta selección y análisis de estudios permite examinar la diversidad de programas de mentoría que existen, así como la pluralidad metodológica presente en la literatura científica. El resumen de la selección de estudios se muestra en un diagrama PRISMA (Figura 1). 
Figura 1. Diagrama PRISMA del proceso de selección de los artículos.

\begin{tabular}{|c|}
\hline Artículos totales $(\mathrm{n}=268)$ \\
\hline Artículos publicados antes del $2010(\mathrm{n}=113)$ \\
\hline < \\
\hline Artículos totales (n=155) \\
\hline Personas $>26$ años $(\mathrm{n}=16)$ \\
\hline ए \\
\hline Artículos totales $(\mathrm{n}=139)$ \\
\hline Objetivos diferentes al análisis de la evaluación de las relaciones ( $\mathrm{n}=77$ ) \\
\hline く々 \\
\hline Artículos totales $(\mathrm{n}=62)$ \\
\hline Duplicados $(\mathrm{n}=6)$ \\
\hline ২ל \\
\hline Artículos totales $(\mathrm{n}=56)$ \\
\hline Métodología no explicita (n=21) \\
\hline$\checkmark \zeta$ \\
\hline Referencias finales $(\mathrm{n}=35)$ \\
\hline
\end{tabular}

Fuente: Elaboración propia.

Treinta y cinco estudios con 164.823 jóvenes $^{1}$ implicados cumplieron los criterios de selección y se incluyeron en el análisis.

\section{RESULTADOS}

Si bien una buena parte de la evidencia empírica revisada procede de evaluaciones de estudios de programas desarrollados en los Estados Unidos (Gráfico 1), siendo éste el país que concentra la mayoría de iniciativas de mentoría de menores y jóvenes; hacer un repaso de ella nos aporta un mayor conocimiento sobre los métodos y técnicas de investigación que se están utilizando. Otros datos de interés que arroja este análisis es que la gran mayoría (87\%) de experiencias de los programas de mentoría son del tipo uno a uno frente a los programas de mentoría grupales (Gráfico 2). También es interesante señalar que del conjunto de personas mentoradas participantes el $60 \%$ fueron hombres y el 40\% mujeres (Gráfico 3).

El 93,6\% de las investigaciones analizadas en esta revisión de la literatura, teniendo en cuenta también los metaanálisis, se basan en métodos cuantitativos (Gráfico 4). Son pocos los estudios que lo hacen desde métodos mixtos o sólo cualitativos aún. De hecho, la mitad de los artículos recogidos utiliza métodos

1 El número total de participantes no incluye las muestras de los estudios de DuBois et al. (2011) y Stumbo et al. (2010), puesto que los autores no especifican la cifra de jóvenes implicados. experimentales en forma de ensayos controlados y aleatorizados. Esta herramienta metodológica puede determinar los mecanismos que en una relación de mentoría conectan causa y efecto si los sujetos de estudio siguen el programa al que han sido asignados al azar. En este caso, es habitual el diseño pretest-postest con grupo de control equivalente, que permite definir y cuantificar los resultados (outcomes) antes y después de haber recibido la mentoría. En esta línea, una de las investigaciones que más tiempo deja transcurrir entre el pre y el postest es la exploración que realizó Rodríguez-Planas (2012) del proyecto Quantum Opportunity —cinco años-, dedicado a fomentar el ingreso a la educación superior de los jóvenes en riesgo de exclusión social. En ésta se pudo observar la eficacia del programa, que aumentó las probabilidades de sus participantes de matricularse en la universidad. Sin embargo, los efectos resultaron ser efímeros y se desvanecieron al cabo de cinco años.

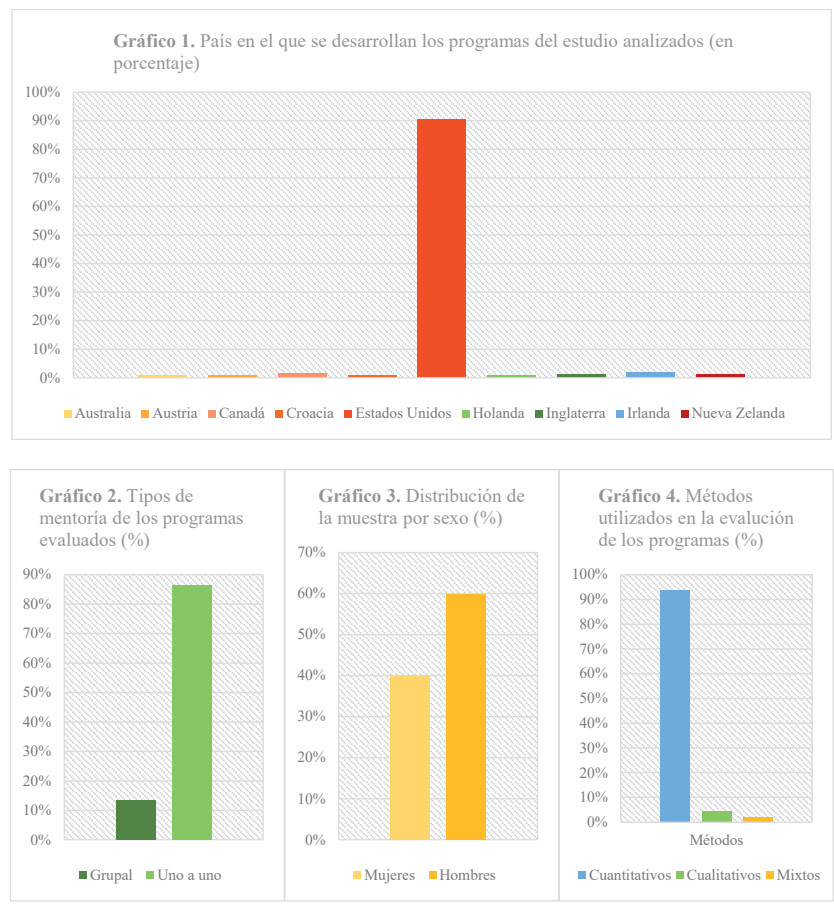

Fuente: Elaboración propia.

La evaluación de la mentoría está gobernada por el diseño pretest-postest, una metodología recurrente en los estudios centrados en el impacto, especialmente cuando se trata de evaluar la eficacia de programas piloto. En el contexto norteamericano, por ejemplo, Curtin et al. (2016) comprobaron la efectividad del proyecto de mentoría The Partners Exploring Education 
and Recreation (PEER), pensado para jóvenes con trastorno del espectro autista, comparando para cada participante las diferencias entre las puntuaciones previas y posteriores a la intervención en un amplio rango de resultados.

Los diseños cuasiexperimentales comparten el mismo propósito que los experimentales: probar la existencia de una relación causal entre dos o más variables. El estudio de Kuperminc, Thomason, DiMeo y Broomfield-Massey (2011) sobre el programa Cool Girls, Inc., enfocado a mejorar el empoderamiento de niñas y adolescentes en situación de vulnerabilidad, utilizó un diseño cuasiexperimental con grupo de control no equivalente para examinar los efectos de la mentoría a corto plazo. Las participantes — 86 en el grupo experimental y 89 en el grupo control-, de entre 9 y 15 años, cumplimentaron un cuestionario antes y después de la intervención de mentoría que medía los niveles de autoconcepto, orientación académica y comportamiento saludable, entre otros. Al comparar las puntuaciones pretest y postest, los autores pudieron observar el impacto que tuvo el apoyo social en las medidas evaluadas.

La homogeneidad en el tratamiento metodológico -imperando lo cuantitativo y, más específicamente, los diseños experimentales basados en ensayos controlados aleatoriamente- se ha ido solventando con la incorporación de nuevas investigaciones cualitativas. En contraste con los métodos experimentales y cuasiexperimentales, los estudios cualitativos tratan de entender el transcurrir de las relaciones y el sentido que toman con el paso del tiempo. En concreto, los estudios basados en entrevistas han contribuido enormemente a nuestra comprensión de las relaciones de mentoría, especialmente de las experiencias negativas. Un ejemplo de ello es la investigación de Spencer, BasualdoDelmonico, Walsh y Drew (2017) sobre cómo y por qué las relaciones de mentoría terminan y de qué manera los participantes experimentan este final. Los autores entrevistaron a 48 parejas del programa BBBSA que acababan de romper. Cada una de las partes fue entrevistada por separado; si bien a los mentores se les contactó por teléfono, a los jóvenes se les citó en sus propias casas ante la presencia de los padres, quienes también ofrecieron su punto de vista. La entrevista semiestructurada abordó aspectos relacionados con el fin del emparejamiento, los motivos y el proceso de ruptura a través de preguntas concretas - como, por ejemplo: «Whose idea was it to end the match?», "What were the reasons for ending the match?» o «How was the ending of the match handled?»- Este trabajo permitió identificar los tipos de ruptura más comunes, las razones que hay detrás y las consecuencias emocionales en toda su complejidad con la riqueza que sólo dan los encuentros cara a cara.

Con una metodología similar, el reciente estudio de Spencer, Gowdy, Drew, McCormack y Keller (2020) aportó un punto de vista complementario: el de la percepción del personal de la agencia BBBSA que realizó el seguimiento de las parejas hasta el fin de su relación. Los autores entrevistaron al personal del programa $(\mathrm{n}=22)$ para saber el rol que desempeñó $\mathrm{y}$ comprobar si ejerció una supervisión correcta de los encuentros; pero también a los mentores $(n=36)$, a los mentorados $(n=36)$ y a los padres de éstos $(n=36)$ con el fin de conocer su impresión acerca de la ruptura, el desarrollo y la calidad de la relación. Las conversaciones telefónicas, que fueron grabadas en audio y transcritas textualmente, duraron hasta 85 minutos y su análisis permitió examinar el proceso dinámico de interacción entre los factores personales y relacionales que condujo a los miembros de la pareja a romper el vínculo.

Algunos de los trabajos empíricos revisados basados en entrevistas han podido documentar no sólo el fin del emparejamiento, sus causas y consecuencias (Spencer et al., 2017, 2020), sino también de qué manera el apoyo social puede desempeñar un papel clave en la vida de los mentorados ayudándoles, por ejemplo, a afrontar situaciones o estresores específicos, como la discriminación racial percibida en la población inmigrante (Griffith, Hurd y Hussain, 2019); o a acceder a nuevas redes de capital social que disminuyan el sentimiento de marginalidad de otros grupos vulnerables como pueden ser las personas con discapacidad (Stewart, Barnfather, Magill-Evans, Ray y Letourneau, 2011) o de sectores urbanos desfavorecidos y bajos ingresos (Sánchez et al., 2011, 2018).

En el contexto europeo, Raithelhuber (2019) realizó entrevistas grupales a menores refugiados no acompañados. Para evitar la sensación de interrogatorio y darle un aire distendido, el autor integró estas entrevistas en actividades socioculturales llevadas a cabo durante un par de reuniones en las que se jugó al futbolín, se escuchó música, se bailó o se fumó. Durante 
cada evento, después de algunas horas de diversión, los participantes fueron invitados a sentarse juntos para empezar con la entrevista. La atmosfera grupal de la charla permitió a los menores refugiados asumir diferentes roles -oyente, orador o comentarista- lo que hizo posible que cada uno pudiera dirigir su propio nivel e intensidad de participación. Este diseño propició una mayor confianza de los menores en el dispositivo y facilitó la comunicación.

La técnica más frecuente en el marco de la investigación cualitativa es la entrevista, aunque otro amplio grupo de trabajos prefiere integrarla en un diseño de métodos múltiples. El estudio de Singh y Tregale (2015) combinó la organización de cinco grupos focales con la realización de entrevistas semiestructuradas a 54 jóvenes de familias refugiadas que participaban en el programa de mentoría LEAP (Learning, Education, Aspiration, Participation) de la Universidad de Macquarie, localizada en Sidney (Australia), donde recibían la ayuda y el apoyo de alumnos del centro que habían vivido experiencias muy parecidas a las suyas. La metodología de discusión permitió explorar la capacidad de impacto de la mentoría entre pares, que ayudó a los jóvenes a mejorar su rendimiento académico y a desarrollar el sentido de pertenencia a la escuela.

El uso de más de un método de investigación ofrece el potencial de un entendimiento más complejo de las relaciones de mentoría, aunque la combinación de estrategias cuantitativas y cualitativas es poco habitual en el campo de la mentoría social. Un ejemplo reciente es el estudio de Dutton, Deane y Bullen (2018) que, además de combinar grupos de discusión con el personal del programa y cuestionarios dirigidos a los participantes, su diseño de investigación concede importancia a otras herramientas como el uso del portfolio, que pone su énfasis en el seguimiento de la relación. Cada portfolio fue elaborado por el mentor, que incluyó todas las actividades que había realizado hasta ese momento con el mentorado, entre otros datos como la información académica o reflexiones personales. Este documento fue clave para esclarecer las respuestas a los cuestionarios y comprobar si el punto de vista de los participantes acerca de la calidad de la mentoría coincidía con la del personal del programa. La compleja interacción entre mentor-mentorado y el resto de personas que rodean a la díada refuerza la importancia de contar con la perspectiva de todos los actores implicados para continuar desarrollando una mejor comprensión de las características de la relación y sus implicaciones para la eficacia de los programas.

Otro de los métodos utilizados en la investigación sobre mentoría es el estudio de casos que, entre otras, emplea técnicas de observación y análisis del discurso con el fin de obtener un mayor entendimiento de la experiencia de los participantes. Aunque su uso es poco habitual, en los últimos años se distingue un creciente interés de los académicos por esta herramienta metodológica en pos de un acercamiento más real del contexto en que los procesos de mentoría cobran sentido. Entre otros trabajos, el estudio de casos de Stumbo et al. (2010) permitió una aproximación a la mentoría de jóvenes con discapacidad, mediante el análisis de cuatro programas financiados por la National Science Foundation que ayudan a los estudiantes a perseguir con éxito una carrera STEM. Los autores realizaron una descripción exhaustiva de cada programa, de su estructura y de las estrategias de reclutamiento de mentores comúnmente empleadas, además de recoger la experiencia de sus participantes con los que mantuvieron algunas charlas. El diseño de investigación con estudios de casos hizo posible para los autores desarrollar un modelo comprensivo sobre el potencial de la mentoría y extraer nuevas reflexiones que sirvieran de guía en el campo de la intervención social, como las ventajas de realizar sesiones online dirigidas a personas con discapacidad.

El estudio de expedientes también ha servido para ampliar el conocimiento que se tiene sobre esquemas menos tradicionales de mentoría donde existe la posibilidad de que los jóvenes se empoderen para tener otras relaciones con adultos de su entorno natural, que refuercen su red de apoyo y puedan actuar como mentores. Este tipo de programas, denominados YouthInitiated Mentoring, han ido creciendo en diferentes países, más allá de los Estados Unidos. En Holanda, por ejemplo, un programa se encarga de este cometido. Durante algo más de medio año, el personal trabaja codo con codo con los participantes y sus familias para escoger aquella persona de su propia red social que les ofrecerá ayuda práctica, consejo y soporte. Para identificar la naturaleza y la gravedad de los problemas de los participantes, Van Dam et al. (2017) analizaron los expedientes de doscientos adolescentes en los que 
se incluía indicaciones de tratamiento, plan familiar y evaluación de diagnóstico. Los autores observaron que el 83\% de los jóvenes estaba preparado para nominar a un mentor informal en tan sólo un mes.

La evidencia empírica demuestra que son muchas y muy variadas las relaciones que se forjan dentro de un mismo programa en el que no todos los participantes logran establecer un vínculo afectivo sólido con su mentor. De ahí la importancia de nuevas aproximaciones que traten de entender cómo y por qué las experiencias de mentoría difieren unas de otras, pudiendo llegar a tener resultados negativos y otras positivos. La etnografía como enfoque permite a un observador externo la posibilidad de participar como testigo mudo de los encuentros, siendo espectador de una cantidad y variedad de situaciones que de otro modo sería imposible. En su estudio, Barrett Cox (2017) recopiló datos a través de la observación participante y entrevistas a diferentes stakeholders - mentores, profesores y administradores - del programa Launch, dirigido a estudiantes de minorías étnicas y de bajos ingresos para hacerles accesible el ingreso a escuelas secundarias de élite. En total, Barrett Cox (2017) pasó más de 500 horas en contacto con los participantes, escribiendo notas de campo diarias, a menudo varias veces durante la jornada, para capturar eventos e interacciones poco después de que ocurrieran.

\section{LIMITACIONES}

La presente revisión de la literatura científica presenta algunas limitaciones. En primer lugar, un reducido número de investigaciones llevadas a cabo fuera de los Estados Unidos. En los últimos años, los programas de mentoría están creciendo de forma importante en muchos países (Preston et al., 2019), pero existen todavía pocos estudios que arrojen algo de luz sobre cuál es el impacto que tiene esta práctica en Europa y qué métodos de evaluación prueban su eficacia. En segundo lugar, debemos remarcar una falta de estudios de alta calidad metodológica. La evaluación de la mentoría está dominada por diseños de investigación que no siempre utilizan el azar para asignar sujetos a tratamientos y por diseños pretest-postest con grupo de control no equivalente. Este tipo de diseño puede verse afectado por diversas amenazas a su validez interna como, por ejemplo, la selección diferencial. Cuando se emplea debe comprobarse si los grupos presentan diferencias estadísticamente significativas en el pretest, y si llega a establecerse una base de comparación apropiada, en aquellas variables que pueden alterar los resultados de eficacia. En tercer lugar, la revisión de la literatura permite observar cómo la diferente exposición de los resultados en los artículos analizados hace imposible aplicar técnicas de metaanálisis clásicas. En muchos de estos artículos la falta de información relevante impide extraer datos fiables para la realización de análisis basados en la razón de razones —en inglés, odds ratio (OR) - o el cálculo de tamaños del efecto necesarios para valorar el impacto de las prácticas asociadas con programas eficaces. Cabe destacar que las variables fundamentales para la estratificación, como la edad, el sexo, la raza o la etnia, no siempre son recogidas. Esto implica que tampoco puedan detectarse la existencia de diferencias en el impacto de la mentoría social de acuerdo a las características individuales de los participantes.

Otra limitación reseñable es la carencia de estudios de seguimiento longitudinales que permitan investigar el desarrollo de los jóvenes como un proceso dinámico enfocado en entender la trayectoria de la relación y no sólo el impacto. La falta de investigaciones que evalúen los resultados de la mentoría a largo plazo impide dar respuesta a una de las preguntas todavía sin resolver en el campo sobre si los efectos de los programas resisten al paso de tiempo, si tienen consecuencias estructurales o bien acaban desapareciendo. Por último, aunque la presente revisión no muestra evidencia de sesgo de publicación, es importante reconocer que los estudios que no respaldan la efectividad de los programas de mentoría podrían ser menos propensos a aparecer en revistas, disertaciones o artículos de investigación revisados por pares lo que podría influir en la interpretación de los resultados.

\section{CONCLUSIONES}

A pesar de que en la actualidad existe un volumen considerable de evidencia robusta que intenta explicar las relaciones de mentoría social, la investigación está dominada por un diseño metodológico experimental basado en pruebas con control aleatorizado que, si bien establece relaciones causa-efecto, puede pasar por alto nuevos conocimientos acerca del proceso y la naturaleza del vínculo forjado entre mentor y mentorado que podrían obtenerse mediante otro tipo de métodos. De 
acuerdo con esto, y en contraste con el uso hegemónico de los ensayos controlados aleatoriamente, este trabajo plantea la pluralidad metodológica como alternativa alentadora en el campo de la mentoría social, aunque se necesitan otros estudios para identificar la forma adecuada de combinar e integrar los métodos cuantitativo y cualitativo. El reto futuro para la investigación de la mentoría consiste en elaborar diseños de métodos mixtos que no sólo aporten un punto de vista relacionado con lo cuantitativo y generalizable, alusivo a patrones que aporten evidencia para la toma de decisiones, sino también que pueda recoger otra dimensión más interpretativa que, considerando la perspectiva subjetiva, ponga la atención en aquello que los jóvenes experimentan en sus encuentros. Esta propuesta metodológica permite comprender el impacto de la mentoría social con un enfoque centrado en los procesos pero también en los resultados.

Además de proponer la necesidad de una metodológica mixta para comprobar el alcance de la mentoría, este artículo subraya la importancia de supervisar los indicadores clave relacionados con la calidad y de disponer de medidas longitudinales para conocer con mayor precisión la efectividad de los programas. Por ejemplo, desde la perspectiva del apoyo social, se entiende como positivo incluir factores externos y contextuales que pueden influir en la mentoría, como la solidaridad cívica o la densidad de entidades cooperativas. A estas posibles líneas de investigación, Raithelhuber (2019b) añade la necesidad de analizar los aspectos sociales y biográficos de los mentores y su impacto en la construcción de la relación de mentoría, cuyos efectos también los atañe. A fin de comprender mejor cómo y por qué algunas relaciones pueden llegar a tener resultados positivos y otras aunque bien intencionadas - pueden resultar dañinas es necesario evaluar la calidad de las díadas, así como del programa al que están sujetas. En este sentido, se destaca la necesidad de contar con un abanico mayor de investigaciones que puedan informar mejor acerca de las nuevas herramientas digitales que, durante los últimos años, están poniendo en marcha cada vez más programas para medir el impacto de la mentoría social a través de la recopilación, gestión y análisis de datos que aportan los participantes. La información que se extrae de estas herramientas es completamente accesible al personal de la agencia y permite, por un lado, el seguimiento de las parejas a efectos de abordar los problemas que puedan darse a medida que progresa la relación; y por otro, la evaluación de la calidad del vínculo establecido entre jóvenes y mentores.

\section{FINANCIACIÓN}

Este artículo ha sido posible con el apoyo de la Secretaria d'Universitats i Recerca del Departament d'Empresa i Coneixement de la Generalitat de Catalunya, de la Unión Europea y del Fondo Social Europeo (FSE) (2021 FI-B2 00160).

\section{AGRADECIMIENTOS}

Estas páginas son parte del Proyecto RECERCAIXA2017UdG, APPlying Mentoring: Innovaciones sociales y tecnológicas para la inclusión social de la población inmigrante y refugiada, financiado por el programa RecerCaixa, una colaboración de la Obra Social "La Caixa" y la Asociación Catalana de Universidades Públicas.

\section{REFERENCIAS BIBLIOGRÁFICAS}

Las referencias marcadas con un asterisco indican estudios comprendidos en la revisión de la literatura científica.

* Barrett Cox, A. (2017). Cohorts, "siblings" and mentors. Organizational structures and the creation of social capital. Sociology of Education, 90(1), 47-63. https://doi. org/10.1177/0038040716681053

* Brady, B., Dolan, P., \& Canavan, J. (2017). 'He told me to calm down and all that': A qualitative study of forms of social support in youth mentoring relationships. Child \& Family Social Work, 22(1), 266-274. https://doi. org/10.1111/cfs.12235

Brady, B., \& O'Regan, C. (2009). Meeting the challenge of doing an RCT evaluation of youth mentoring in Ireland: A journey in mixed methods. Journal of Mixed Methods Research, 3(3), 265-280. https://doi. org/10.1177/1558689809335973

Brumovská, T., \& Seidlová Málková, G. (2020). The methodological issues in the assessment of the quality and benefits of formal youth mentoring interventions: The case of the Czech Big Brothers Big Sisters/Pět P. En Ò. Prieto-Flores y J. Feu (Eds.), Mentoring Children and Young People for Social Inclusion: Global Approaches to Empowerment (pp. 110-127). London: Routledge.

* Ciocanel, O., Power, K., Eriksen, A., \& Gillings, K. (2017). Effectiveness of positive youth development 
interventions: A meta-analysis of randomized controlled trials. Journal of Youth and Adolescence, 46, 483-504. https://doi.org/10.1007/s10964-016-0555-6

* Curtin, C., Humphrey, K., Vronsky, K., Mattern, K., Nicastro, S., \& Perrin, E.C. (2016). Expanding horizons: a pilot mentoring program linking college/graduate students and teens with ASD. Clinical Pediatrics, 55(2), 150-156. https://doi.org/10.1177/0009922815588821

Deutsch, N., \& Spencer, R. (2009). Capturing the magic: assessing the quality of youth mentoring relationships. New Directions for Youth Development: Theory, Practice and Research, 121, 47-70. https://doi.org/10.1002/yd.296

* Dolan, P., Brady, B., O'Regan, C., Canavan, J., Russell, D., \& Forkan, C. (2011). Big Brothers Big Sisters (BBBS) of Ireland: Evaluation study. Report 1: Randomised Controlled Trial and Implementation Report: UNESCO Child and Family Research Centre on behalf of Foróige: Galway, Ireland

DuBois, D. L., Holloway, B. E., Valentine, J. C., \& Cooper, H. (2002). Effectiveness of mentoring programs for youth: A meta-analytic review. American Journal of Community Psychology, 30(2), 157-197. https://doi. org/10.1023/A:1014628810714

DuBois, D. L., \& Karcher, M. J. (2005). Youth Mentoring: Theory, Research and Practice. En D. L. DuBois \& M. J. Karcher (Eds.), Handbook of youth mentoring (2-11). Thousand Oaks, CA: Sage.

* DuBois, D. L., Portillo, N., Rhodes, J. E., Silverthorn, N., \& Valentine, J. C. (2011). How effective are mentoring programs for youth? A systematic assessment of the evidence. Psychological Science in the Public Interest, 12(2), 57-91. https://doi.org/10.1177/1529100611414806

DuBois, D. L., \& Silverthorn N. (2005). Natural mentoring relationships and adolescent health: evidence from a national study. American Journal of Public Health, 95(3), 518-525. https://doi.org/10.2105/AJPH.2003.031476

* Dutton, H., Deane, K. L., \& Bullen, P. (2018). Distal and experiential perspectives of relationship quality from mentors, mentees, and program staff in a schoolbased youth mentoring program. Children and Youth Services Review, 85, 53-62. https://doi.org/10.1016/j. childyouth.2017.12.008

* Erdem, G., DuBois, D. L., Larose, S., De Wit, D., \& Lipman, E. L. (2016). Mentoring relationships, positive development, youth emotional and behavioral problems: Investigation of a mediational model. Journal of Community Psychology, 44(4), 464-483. https://doi. org/10.1002/jcop.21782

* Griffith, A. N., Hurd, N. M., \& Hussain, S. B. (2019) "I didn't come to school for this": A qualitative examination of experiences with race-related stressors and coping responses among black students attending a predominantly white institution. Journal of Adolescent Research, 34(2) 115-139. https://doi. org/10.1177/0743558417742983

Harris, J. T., \& Nakkula, M. J. (2018). Match Characteristics Questionnaire (MCQ) [Unpublished measure]. Applied Research Consulting.
* Hurd, N. M., Sánchez, B., Zimmerman, M. A., \& Caldwell, C. H. (2012). Natural mentors, racial identity, and educational attainment among African American adolescents: Exploring pathways to success. Child Development, 83(4), 1196-1212. https://doi.org/10.1111/ j.1467-8624.2012.01769.x

* Kanchewa, S. S., Rhodes, J. E., Schwartz, S. E. O., \& Olsho, L. E. W. (2014). An investigation of same- versus cross-gender matching for boys in formal school-based mentoring programs. Applied Developmental Science, 18(1), 31-45. https://doi.org/10.1080/10888691.2014.876251

* Kolakowsky-Hayner, S. A., Wright, J., Shem, K., Medel, R., \& Duong, T. (2012). An effective community-based mentoring program for return to work and school after brain and spinal cord injury. NeuroRehabilitation, 31(1), 63-73. https://doi.org/10.3233/NRE-2012-0775

* Kuperminc, G. P., Thomason, J., DiMeo, M., \& BroomfieldMassey, K. (2011). Cool Girls, Inc.: Promoting the positive development of urban preadolescent and early adolescent girls. The Journal of Primary Prevention, 32, 171-183. https://doi.org/10.1007/s10935-011-0243-y

* Kupersmidt, J. B., Stump, K. N., Stelter, R. L., \& Rhodes, J. E. (2017). Mentoring program practices as predictors of match longevity. Journal of Community Psychology, 45(5), 630-645. https://doi.org/10.1002/jcop.21883

* Liao, C. L., \& Sánchez, B. (2015). An exploratory study of the role of mentoring in the acculturation of Latino/a youth. Journal of Community Psychology, 43(7), 868-877. https://doi.org/10.1002/jcop.21717

* Liao, C. L., \& Sánchez, B. (2019). Mentoring relationship quality profiles and their association with urban, lowincome youth's academic outcomes. Youth E Society, 51 (4), 443-462. https://doi.org/10.1177/0044118X16668058

* Marino, C., Santinello, M., Lenzi, M., Santoro, P., Bergamin, M., Gaboardi, M., ... \& Perkins, D. D. (2019). Can mentoring promote self-esteem and school connectedness? An evaluation of the Mentor-UP Project. Psychosocial Intervention, 29(1), 1-8. https://doi. org/10.5093/pi2019a13

Pickering, C., \& Byrne, J. (2013). The benefits of publishing systematic quantitative literature reviews for $\mathrm{PhD}$ candidates and other early-career researchers. Higher Education Research \& Development, 33(3), 534-548. https:// doi.org/10.1080/07294360.2013.841651

Preston, J. M., Prieto-Flores, Ò., \& Rhodes, J. E. (2019). Mentoring in context: A comparative study of youth mentoring programs in the United States and continental Europe. Youth \& Society, 51(7), 900-914. https://doi. org/10.1177/0044118X18813700

Prieto-Flores, Ò., y Feu, J. (2018). ¿Qué impacto pueden tener los programas de mentoría social en la sociedad? Una exploración de las evaluaciones existentes y propuesta de marco analítico. Pedagogía Social. Revista Interuniversitaria, 31, 153-167. https://doi.org/10.7179/ PSRI_2018.31.12

Pryce, J., Deane, K. L., Barry, J. E., \& Keller, T. E. (2020). Understanding youth mentoring relationships: Advancing the field with direct observational methods. 
Adolescent Research Review, 1-12. https://doi.org/10.1007/ s40894-019-00131-z

* Raithelhuber, E. (2019a). 'If we want, they help us in any way': How 'unaccompanied refugee minors' experience mentoring relationships. European Journal of Social Work, (n/s). https://doi.org/10.1080/13691457.2019.1606787

Raithelhuber, E. (2019b). Turning into a "Godparent": How adult volunteers negotiate their personal life to become a mentor for "Unaccompanied Refugee Minors". Social Work and Social Welfare, 1(1): 23-36. https://doi. org/10.25082/SWSW.2019.01.003

* Raposa, E. B., Ben-Eliyahu, A., Olsho, L. E. W., \& Rhodes, J. E. (2018). Birds of a feather: Is matching based on shared interests and characteristics associated with longer youth mentoring relationships? Journal of Community Psychology, 47(2), 385-397. https://doi.org/10.1002/jcop. 22127

Raposa, E. B., Dietz, N., \& Rhodes, J. E. (2017). Trends in volunteer mentoring in the United States: Analysis of a decade of Census Survey Data. American Journal of Community Psychology, 59, 3-14. https://doi.org/10.1002/ ajcp. 12117

* Raposa, E. B., Rhodes, J. E., Stams, G. J. J. M., Card, N., Burton, S., Schwartz, S., ... \& Hussain, S. (2019). The effects of youth mentoring programs: A meta-analysis of outcome studies. Journal of Youth and Adolescence, 48, 423-443. https://doi.org/10.1007/s10964-019-00982-8

Rhodes, J. E., Reddy, R., Roffman, J., \& Grossman, J. (2005). Promoting successful youth mentoring relationships: A preliminary screening questionnaire. Journal of Primary Prevention, 26, 147-167. https://doi.org/10.1007/s10935005-1849-8

Rhodes, J. E., Schwartz, S. E. O., Willis, M. M., \& Wu, M. B. (2017). Validating a mentoring relationship quality scale: Does match strength predict match length? Youth \& Society, 49(4), 415-437. https://doi. org/10.1177/0044118X14531604

* Rodríguez-Planas, N. (2012). Longer-term impacts of mentoring, educational services, and learning initiatives: evidence from a randomized trial in the United States. American Economic Journal: Applied Economics, 4(4), 121139. https://doi.org/10.1257/app.4.4.121

* Sánchez, B., Esparza, P., Berardi, L., \& Pryce, J. (2011). Mentoring in the context of Latino youth's broader village during their transition from high school. Youth and Society, 43(1), 225-252. https://doi. org/10.1177/0044118X10363774

* Sánchez, B., Pinkston, K. D., Cooper, A. C., Luna, C., \& Wyatt, S. T. (2018). One falls, we all fall: How boys of color develop close peer mentoring relationships. Applied Developmental Science, 22(1), 14-28. https://doi.org/10.10 80/10888691.2016.1208092

* Schwartz, S. E. O., Rhodes, J. E., Spencer, R., \& Grossman, J. B. (2013). Youth initiated mentoring: Investigating a new approach to working with vulnerable adolescents. American Journal of Community Psychology, 52(1-2), 155169. https://doi.org/10.1007/s10464-013-9585-3

* Silke, C., Brady, B., \& Dolan, P. (2019). Relational dynamics in formal youth mentoring programs: A longitudinal investigation into the association between relationship satisfaction and youth outcomes. Children and Youth Services Review, 104, 104343. https://doi.org/10.1016/j. childyouth.2019.05.020

* Singh, S., \& Tregale, R. (2015). From homeland to home: Widening participation through the LEAP-Macquarie mentoring (refugee mentoring) program. International Studies in Widening Participation, 2(1), 15-27.

* Spencer, R., Basualdo-Delmonico, A., Walsh, J., \& Drew, A.L. (2017). Breaking up is hard to do: A qualitative interview study of how and why youth mentoring relationships end. Youth \& Society, 49(4), 438-460. https:// doi.org/10.1177/0044118X14535416

* Spencer, R., Drew, A. L., Walsh, J., \& Kanchewa, S. S. (2018). Girls (and Boys) Just want to have fun: A mixed-methods examination of the role of gender in youth mentoring relationship duration and quality. The Journal of Primary Prevention. 39(1), 17-35. https://doi.org/10.1007/s10935017-0494-3

* Spencer, R., Gowdy, G., Drew, A. L., McCormack, M. J., \& Keller, T. E. (2020). It takes a village to break up a match: A systemic analysis of formal youth mentoring relationship endings. Child \& Youth Care Forum, 49(1), 97120. https://doi.org/10.1007/s10566-019-09520-w

* Stewart, M., Barnfather, A., Magill-Evans, J., Ray, L., \& Letourneau, N. (2011). Brief report: An online support intervention: perceptions of adolescents with physical disabilities. Journal of Adolescence, 34(4), 795-800. https:// doi.org/10.1016/j.adolescence.2010.04.007

* Stumbo, N., Martin, J. K., Nordstrom, D., Rolfe, T., Burgstahler, S., Whitney, J., ... \& Misquez, E. (2010). Evidence-based practices in mentoring students with disabilities: Four case studies. Journal of Science Education for Students with Disabilities, 14(1), 33-54. https://doi. org/10.14448/jsesd.03.0003

Tierney, J. P., Grossman, J. B., \& Resch, N. L. (1995). Making a Difference: An Impact Study of Big Brothers Big Sisters. Philadelphia: Public/Private Ventures.

* Van Dam, L., Smit, D., Wildschut, B., Branje, S. J. T., Rhodes, J. E., Assink, M., \& Stams, G. J. J. M. (2018). Does natural mentoring matter: A multilevel meta-analysis on the association between natural mentoring and youth outcomes. American Journal of Community Psychology, 62(12), 203-220. https://doi.org/10.1002/ajcp.12248

* Van Dam, L., Neels, S., De Winter, M., Branje, S., Wijsbroek, S., Hutschemaekers, G., ... \& Stams, G. J. J. M. (2017). Youth initiated mentors: do they offer an alternative for out-of-home placement in youth care? The British Journal of Social Work, 47(6) 1764-1780. https://doi.org/10.1093/ bjsw/bcx092

* Wittrup, A. R., Hussain, S. B., Albright, J. N., Hurd, N. M., Varner, F. A., \& Mattis, J. S. (2019). Natural mentors, racial pride, and academic engagement among black adolescents: Resilience in the context of perceived discrimination. Youth \& Society, 51(4), 463-483. https:// doi.org/10.1177/0044118X16680546

* Wood, S., \& Mayo-Wilson, E. (2012). School-based mentoring for adolescents: A systematic review and 
meta-analysis. Research on Social Work Practice, 22(3), 257-269. https://doi.org/10.1177/1049731511430836

Zand, D. H., Thomson, N., Cervantes, R., Espiritu, R., Klagholz, D., LaBlanc, L., \& Taylor, A. (2009). The mentor-youth alliance: The role of mentoring relationships in promoting youth competence. Journal of Adolescence, 32, 1-17. https://doi.org/10.1016/j. adolescence.2007.12.006

\section{NOTAS BIOGRÁFICAS}

\section{ANNA SÁNCHEZ-ARAGÓN}

Graduada en Periodismo por la Universidad Rovira i Virgili y Máster Oficial en Estudios de Género y Gestión de Políticas de Igualdad por la Universidad de Lleida. En la actualidad, es doctoranda en el programa de Estudios Humanísticos de la URV y miembro del grupo de investigación Social $\&$ Business Research Laboratory. Sus principales líneas de investigación giran en torno a la igualdad y la mentoría social.

\section{ANGEL BELZUNEGUI-ERASO}

Doctor en Sociología por la Universidad Autónoma de Barcelona. Director de la Cátedra de Inclusión Social de la Universidad Rovira i Virgili donde es profesor titular. Ha sido coordinador del Módulo Jean Monnet titulado «The challenges of the European Union in the social agenda: poverty, inequality and social inclusion», financiado por la UE. Es coordinador, junto al Dr. Luis Moreno, de la obra colectiva La Agenda Social Europea. Cohesión Social y Lucha contra las Desigualdades en Europa, publicada en Thomson-Aranzadi (2020).

\section{ÒSCAR PRIETO-FLORES}

Doctor en Sociología por la Universidad de Barcelona. Profesor agregado del Departamento de Pedagogía de la Universidad de Girona. Investigador principal del proyecto RecerCaixa «APPlying Mentoring: Innovaciones sociales y tecnológicas para la inclusión de la personas inmigrantes y refugiadas»(2018-2021). Su último libro ha sido publicado recientemente en Routledge Mentoring Children and Young people for Social Inclusion: Global Approaches to Empowerment (2020). 


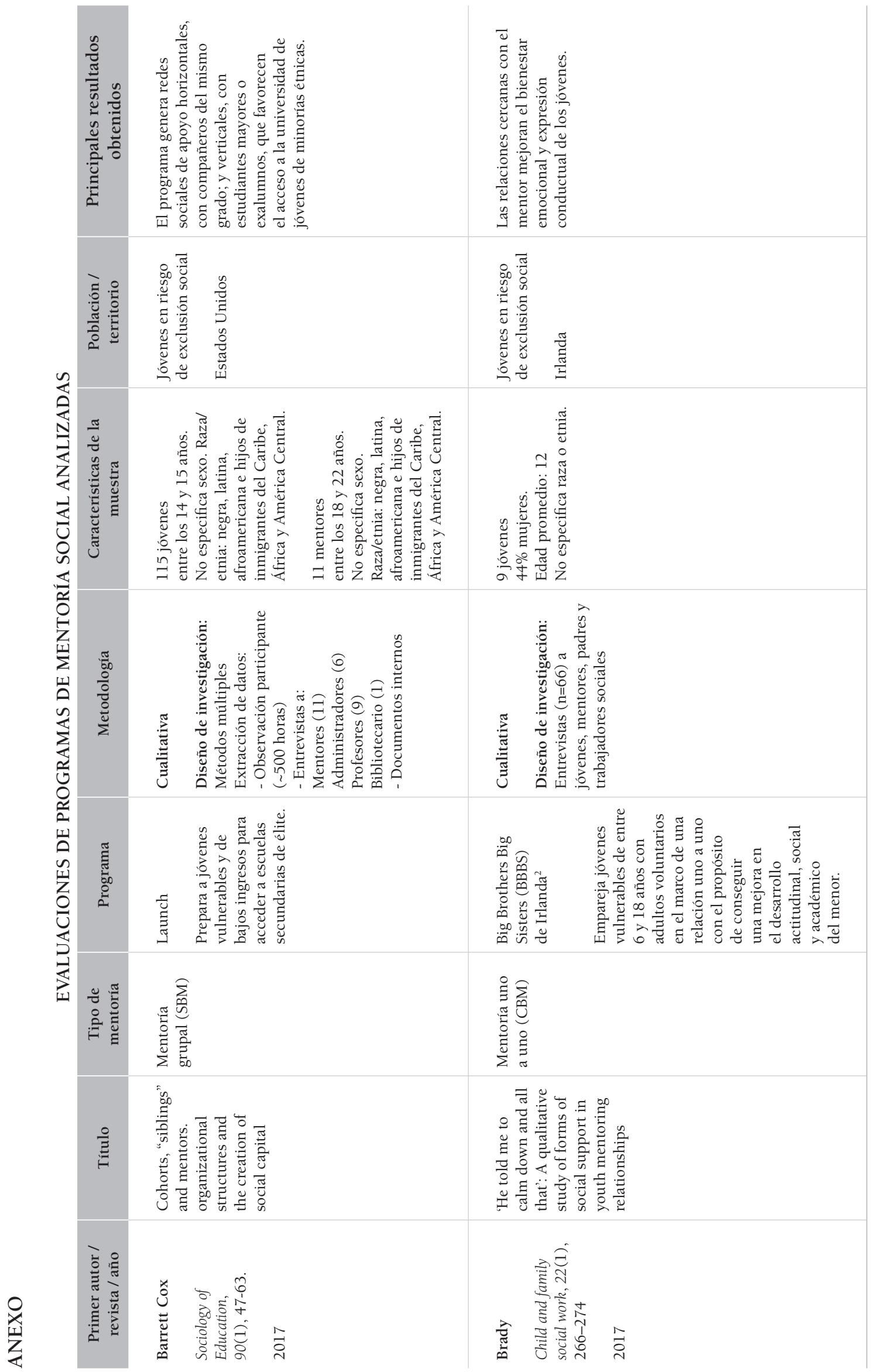




\begin{tabular}{|c|c|c|c|}
\hline 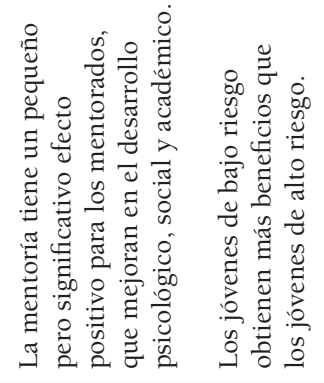 & 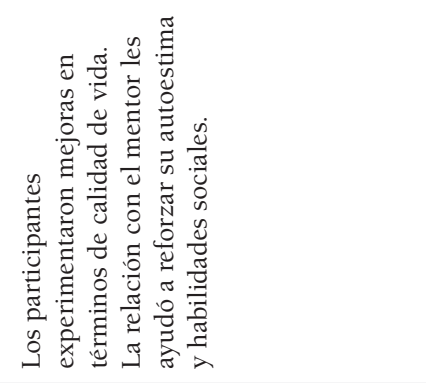 & 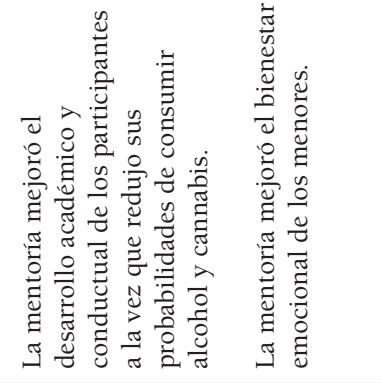 & 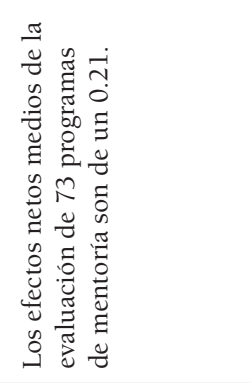 \\
\hline 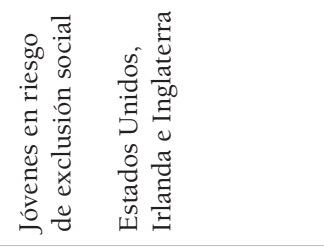 & 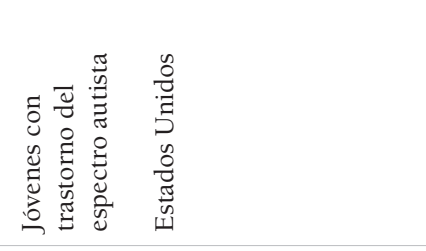 & 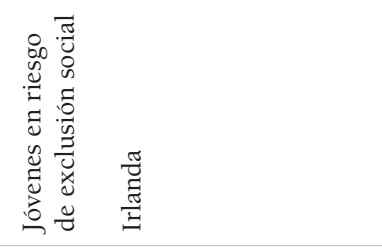 & 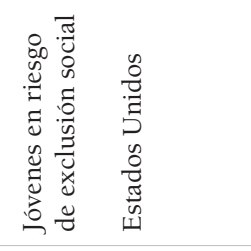 \\
\hline 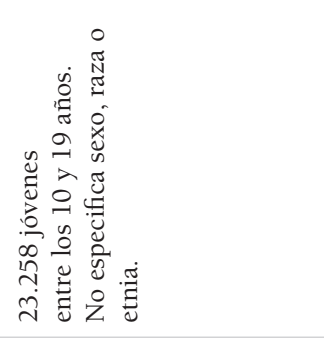 & 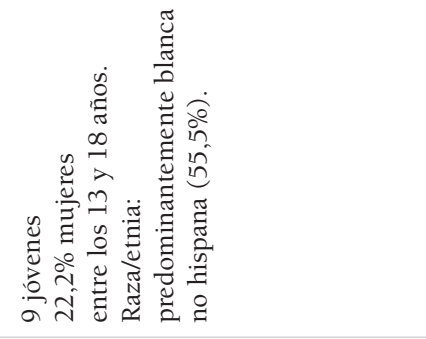 & 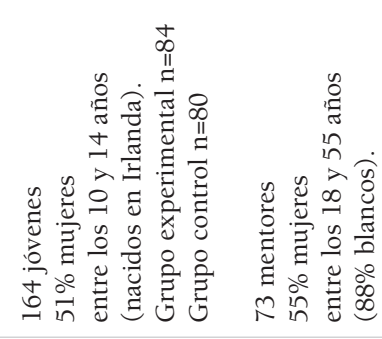 & 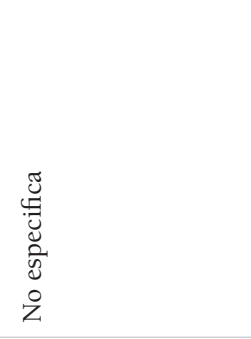 \\
\hline 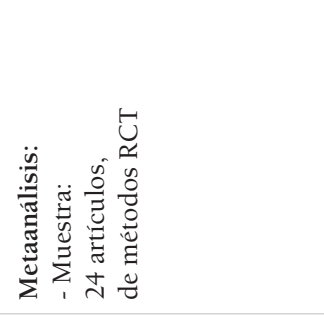 & 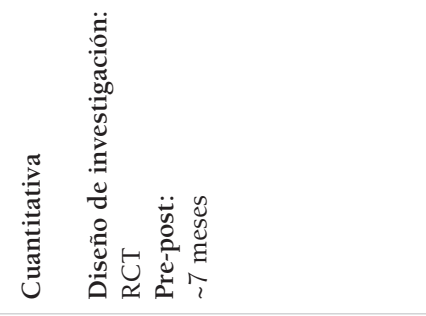 & 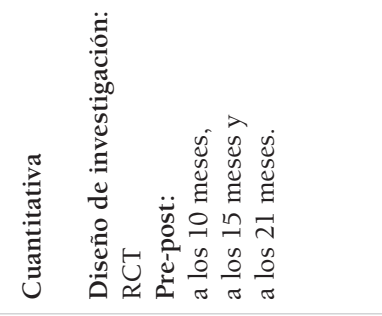 & 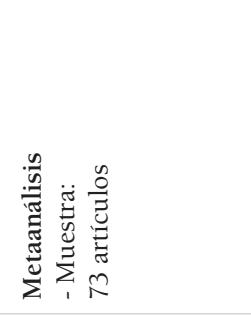 \\
\hline 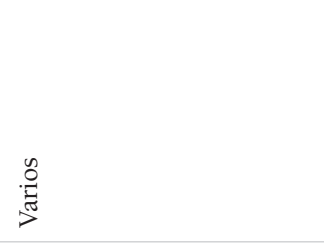 & 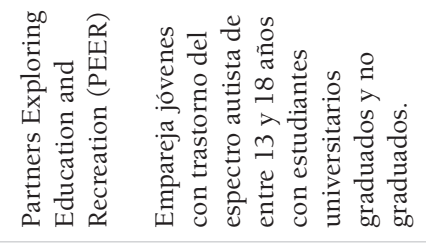 & 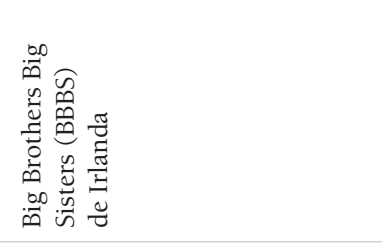 & 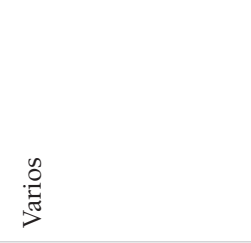 \\
\hline 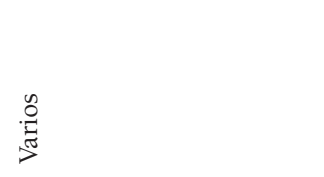 & 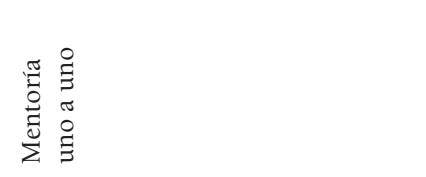 & 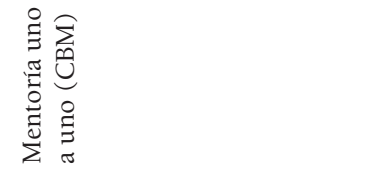 & 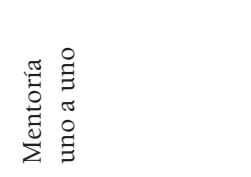 \\
\hline 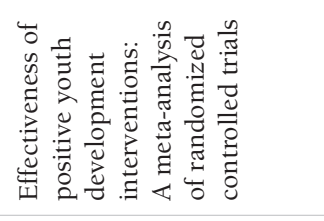 & 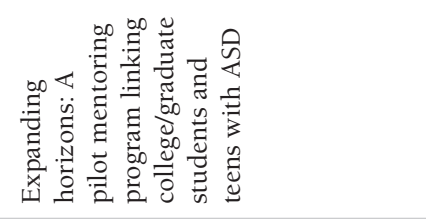 & 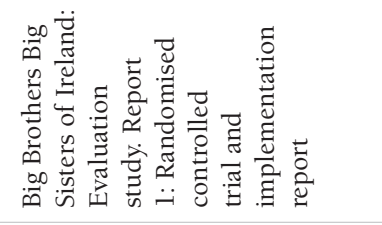 & 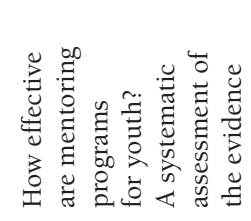 \\
\hline 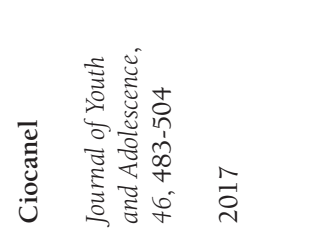 & 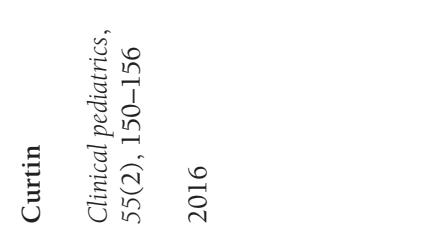 & 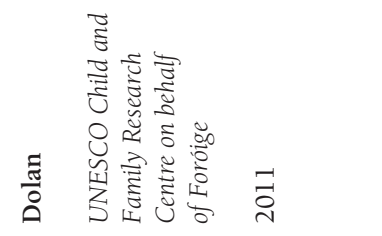 & 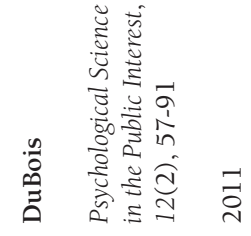 \\
\hline
\end{tabular}




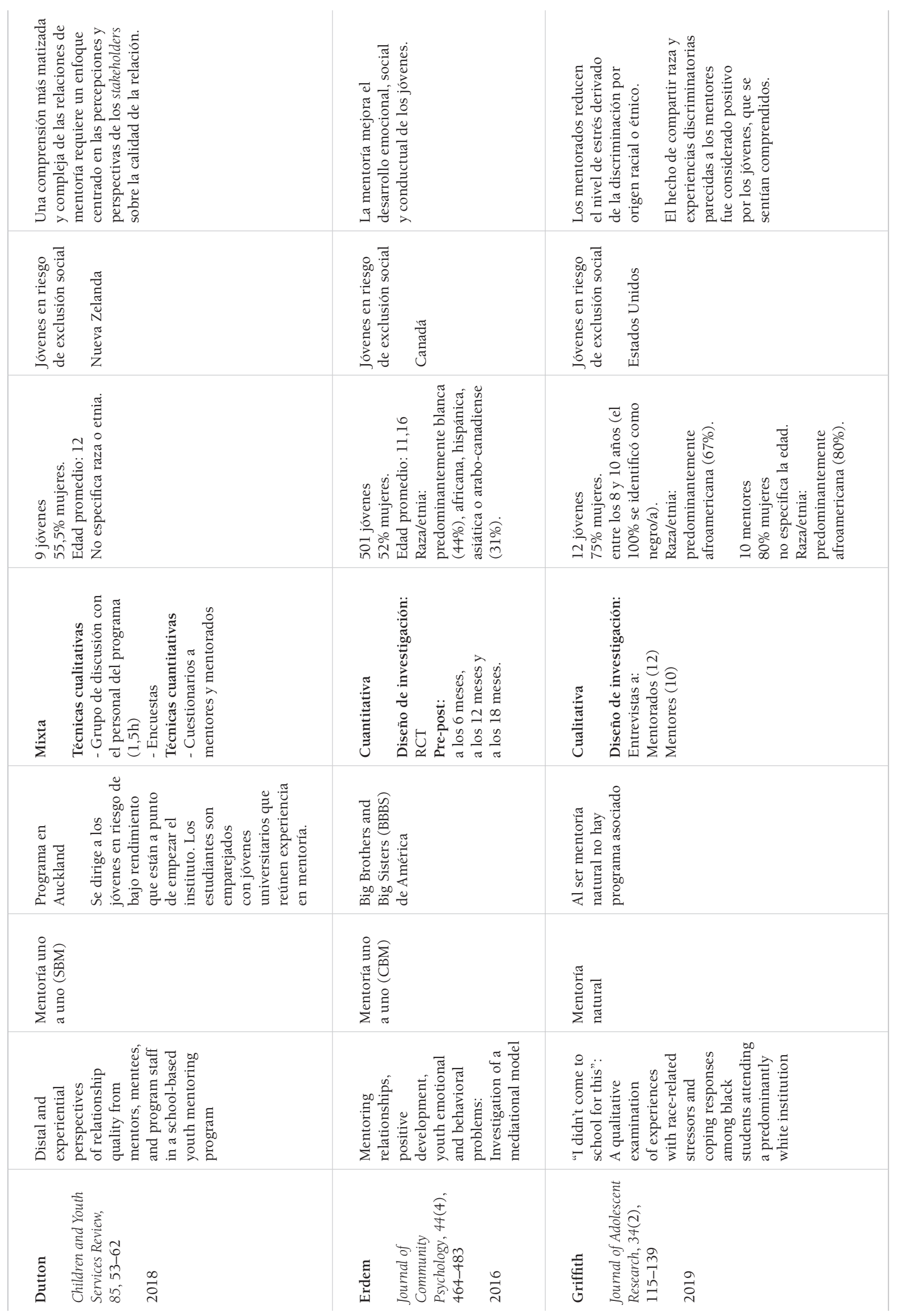




\begin{tabular}{|c|c|c|}
\hline 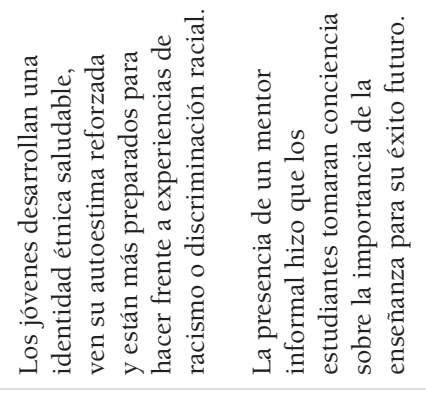 & 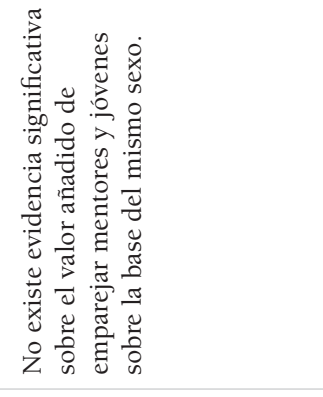 & 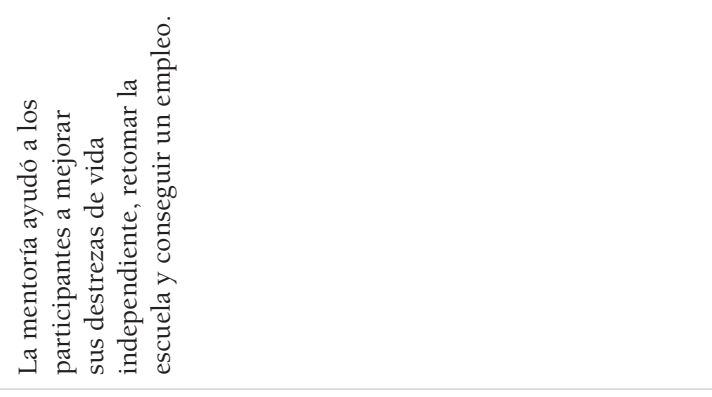 \\
\hline 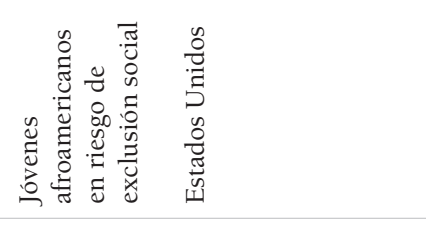 & 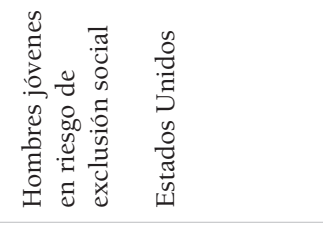 & 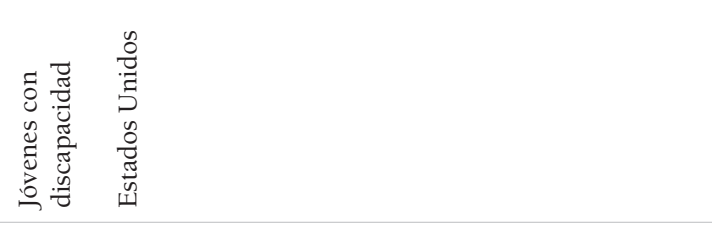 \\
\hline 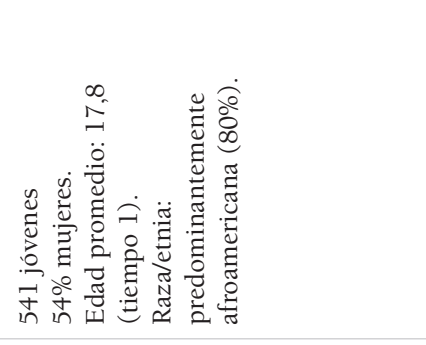 & 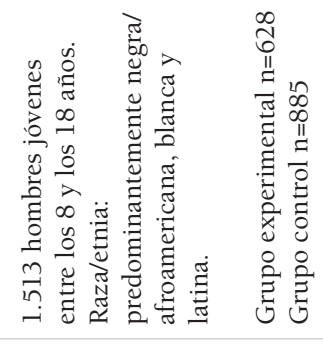 & 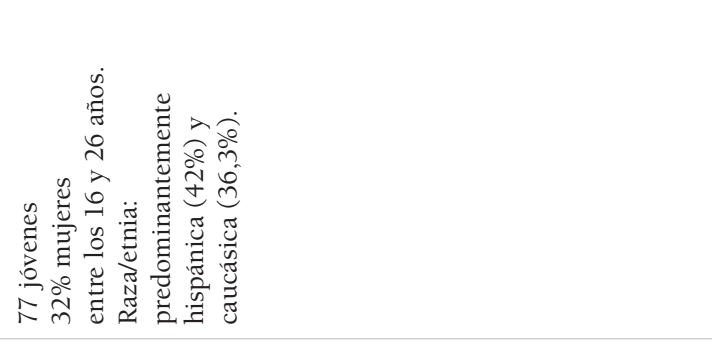 \\
\hline 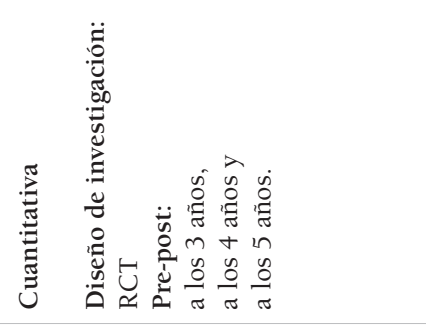 & 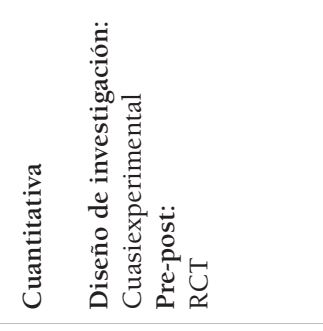 & 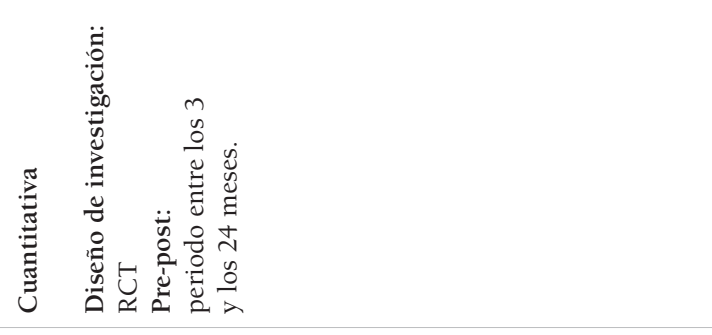 \\
\hline 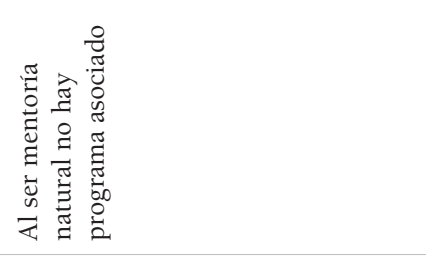 & 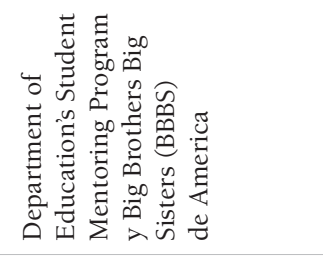 & 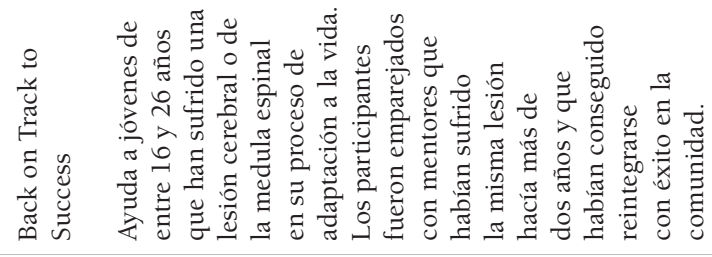 \\
\hline 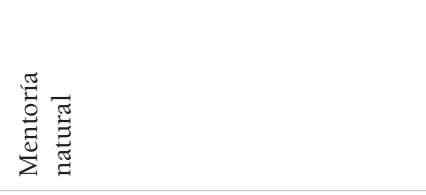 & 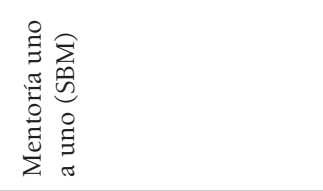 & 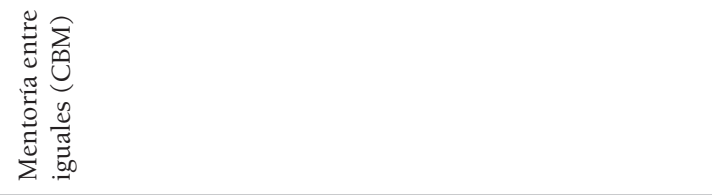 \\
\hline 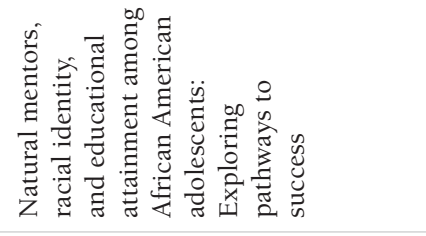 & 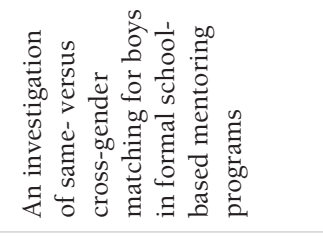 & 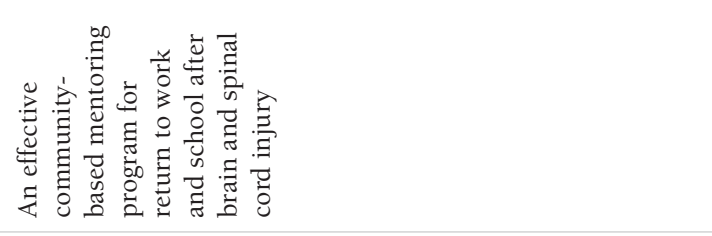 \\
\hline 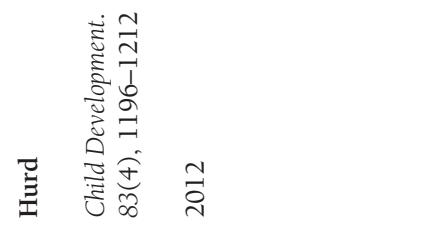 & 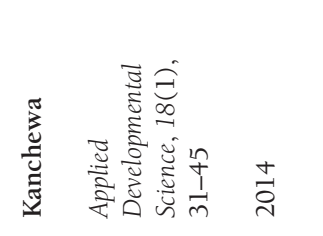 & 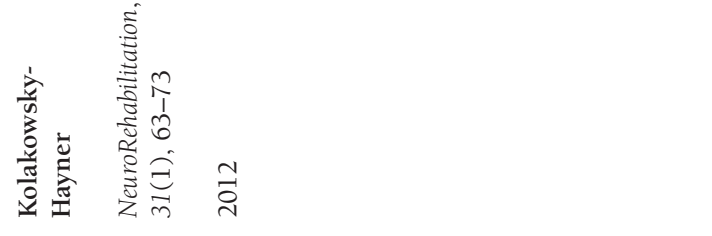 \\
\hline
\end{tabular}




\begin{tabular}{|c|c|c|}
\hline 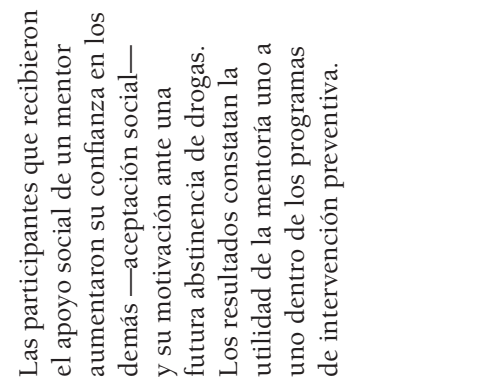 & 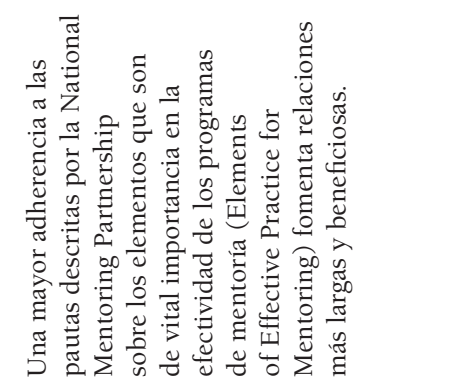 & 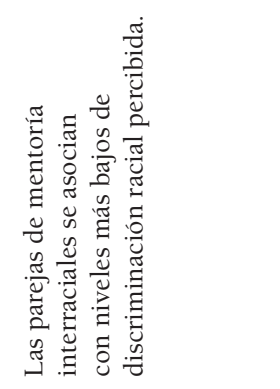 \\
\hline 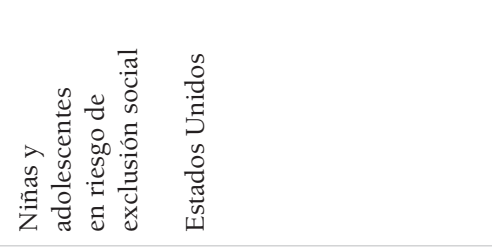 & 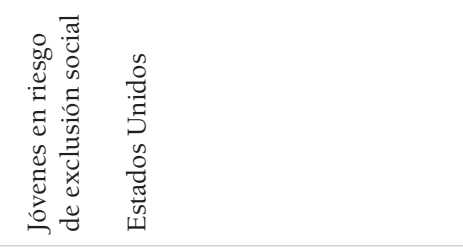 & 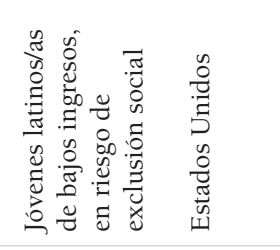 \\
\hline 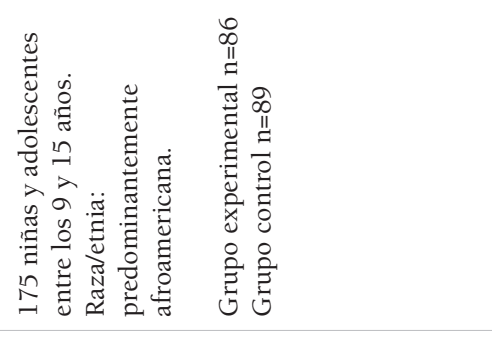 & 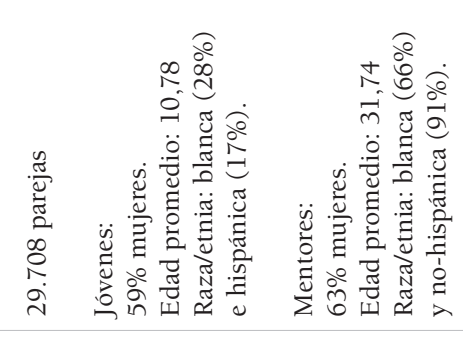 & 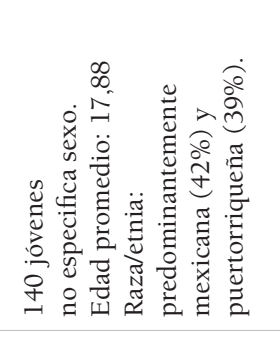 \\
\hline 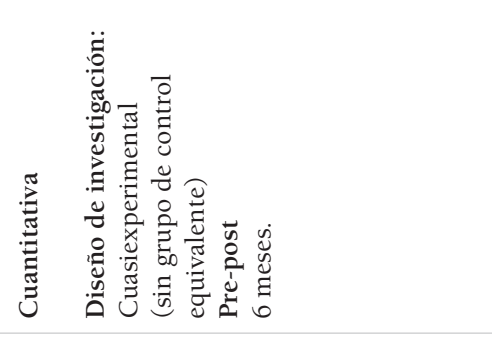 & 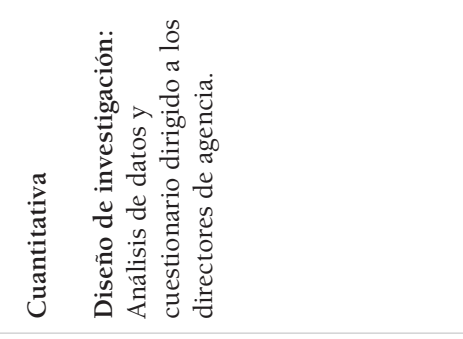 & 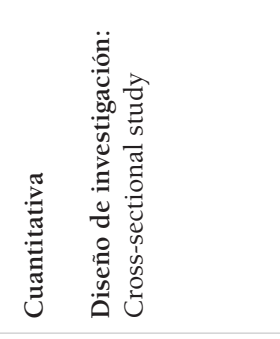 \\
\hline 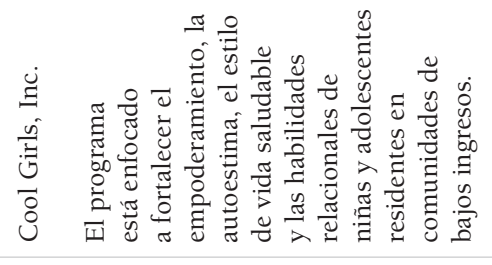 & 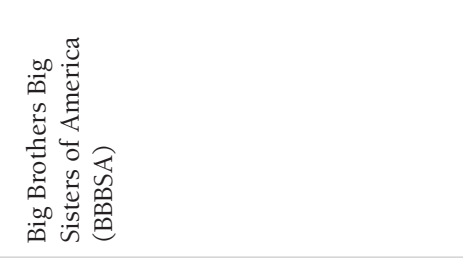 & 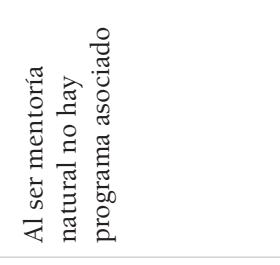 \\
\hline 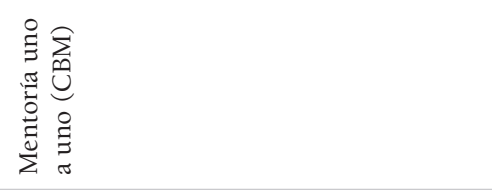 & 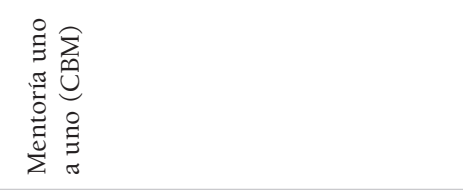 & 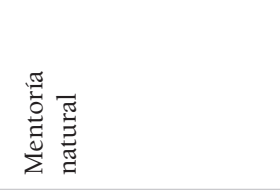 \\
\hline 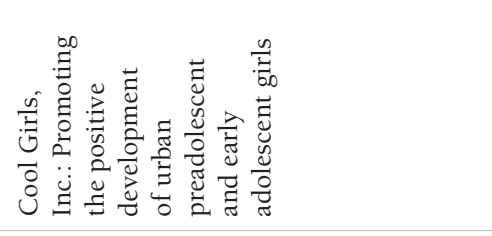 & 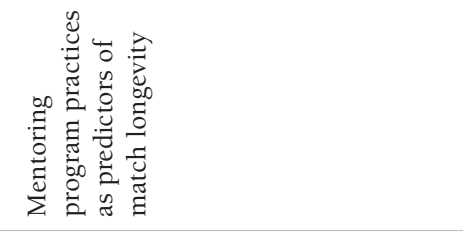 & 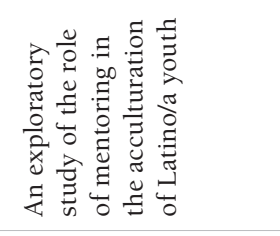 \\
\hline 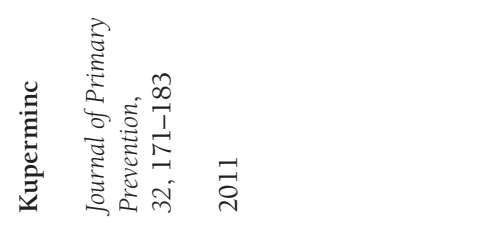 & 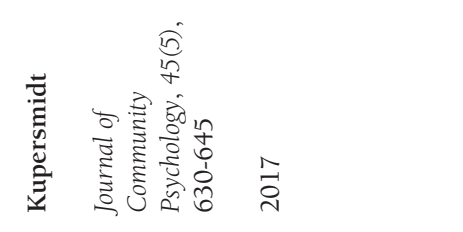 & 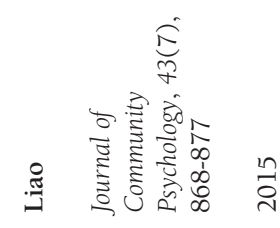 \\
\hline
\end{tabular}




\begin{tabular}{|c|c|c|}
\hline 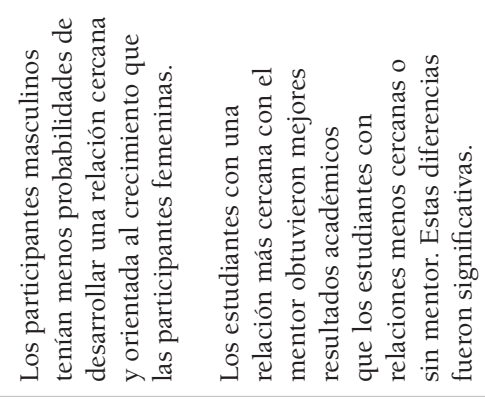 & 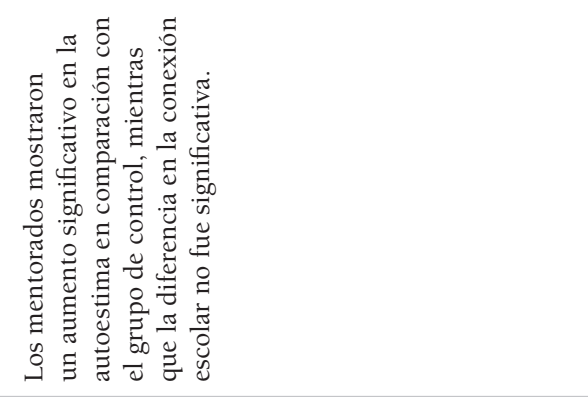 & 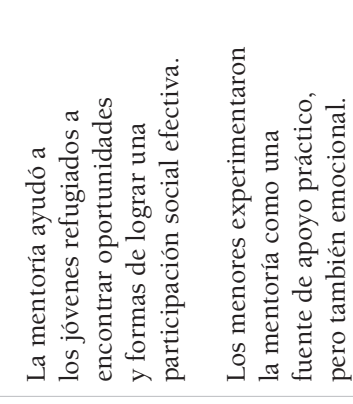 \\
\hline 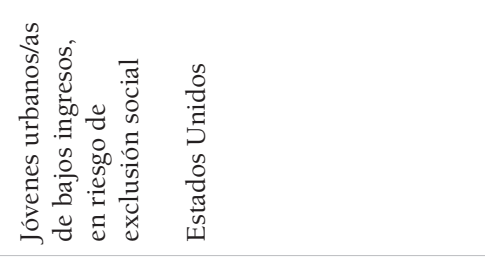 & 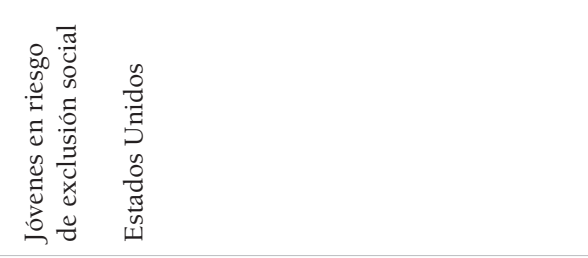 & 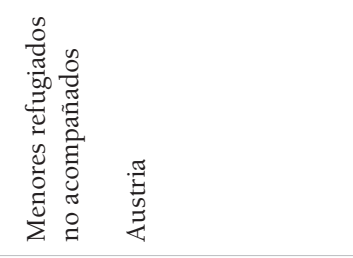 \\
\hline 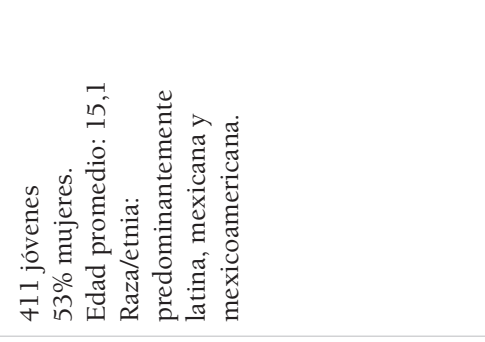 & 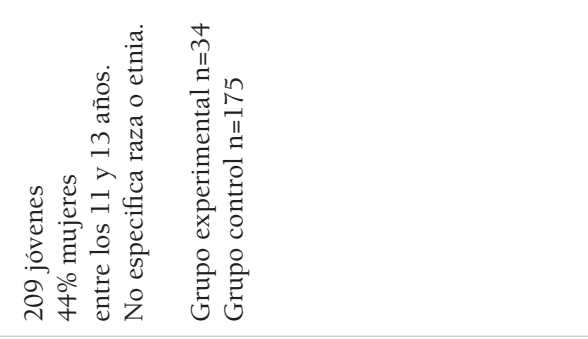 & 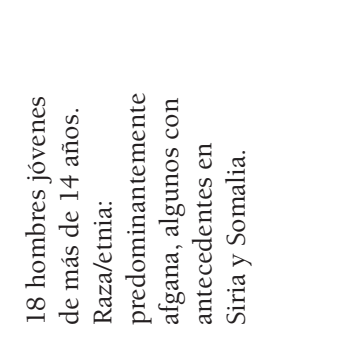 \\
\hline 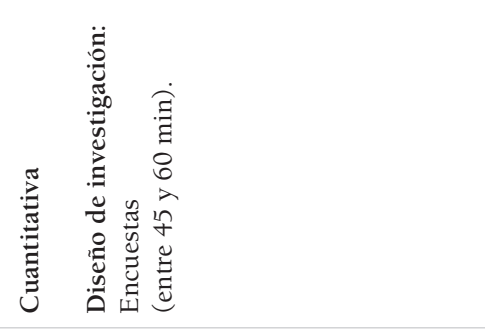 & 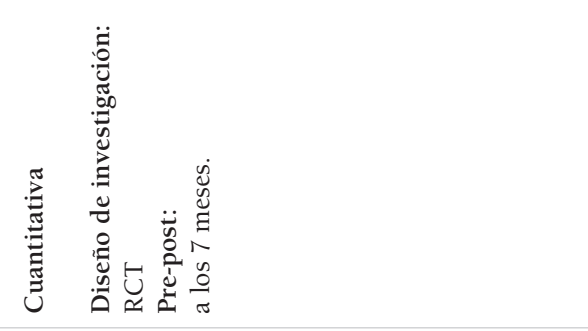 & 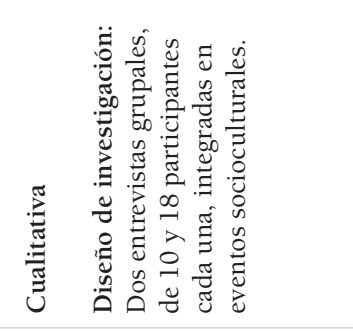 \\
\hline 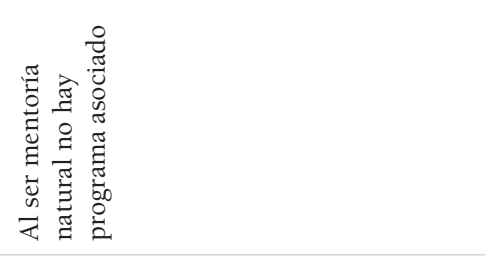 & 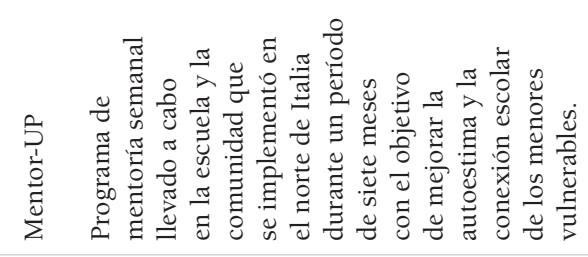 & 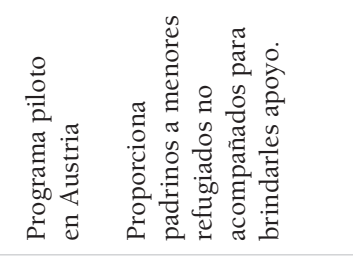 \\
\hline 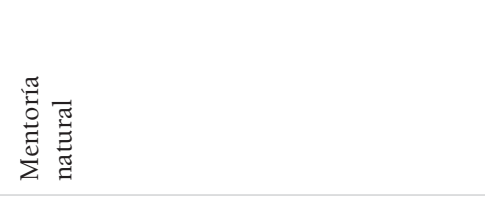 & 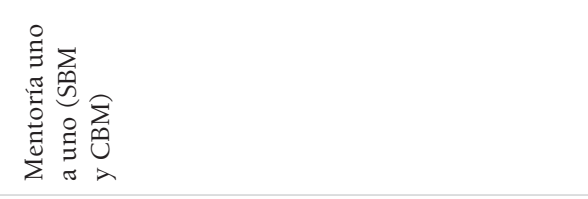 & 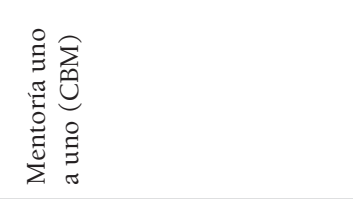 \\
\hline 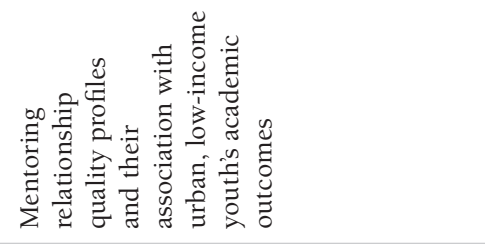 & 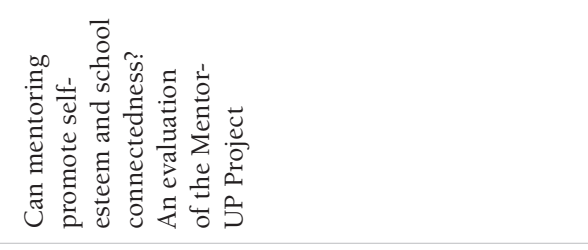 & 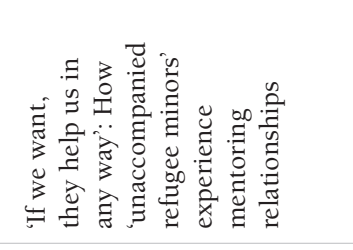 \\
\hline 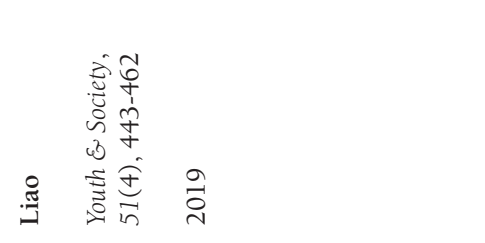 & 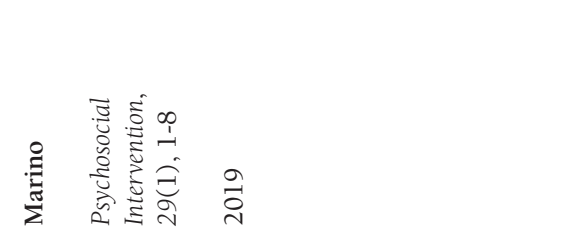 & 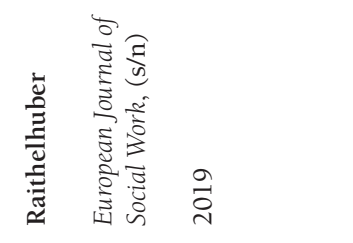 \\
\hline
\end{tabular}




\begin{tabular}{|c|c|c|c|}
\hline 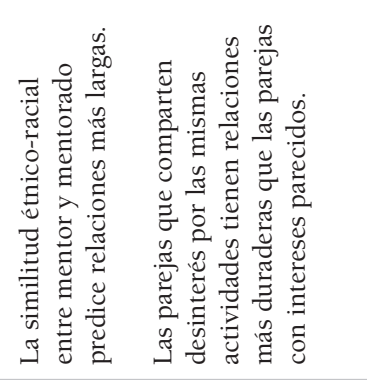 & & 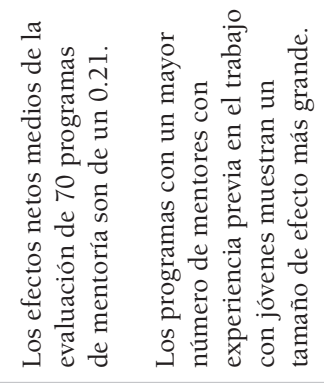 & 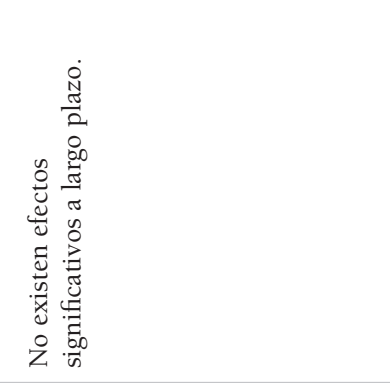 \\
\hline 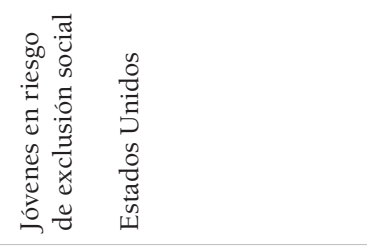 & & 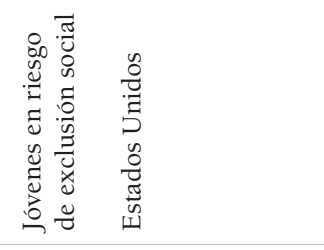 & 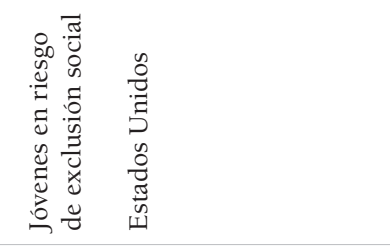 \\
\hline 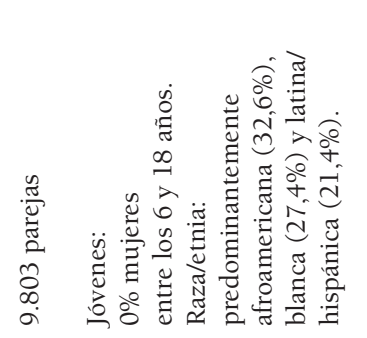 & 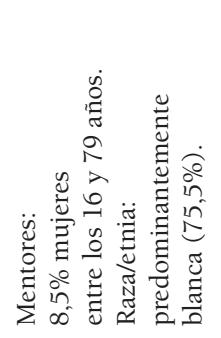 & 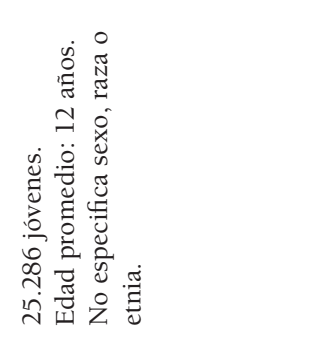 & 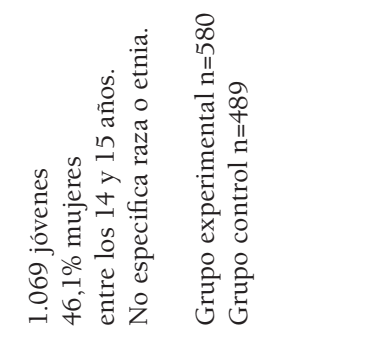 \\
\hline 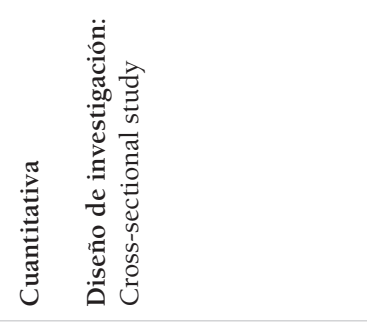 & & 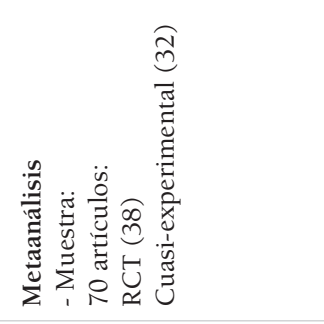 & 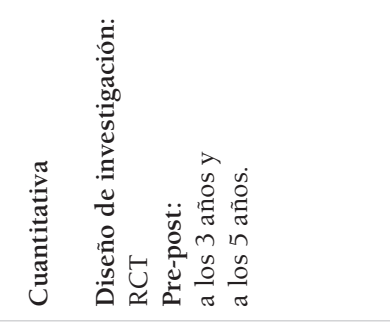 \\
\hline 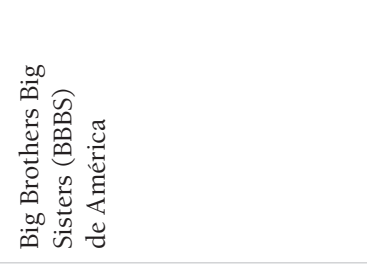 & & 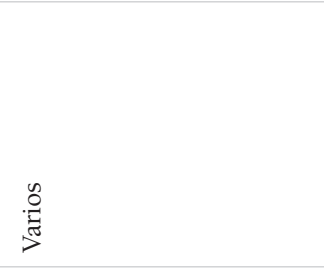 & 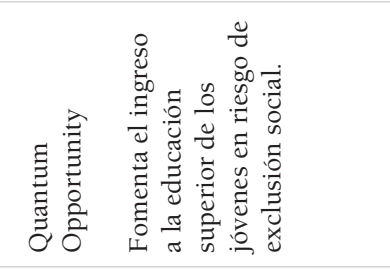 \\
\hline 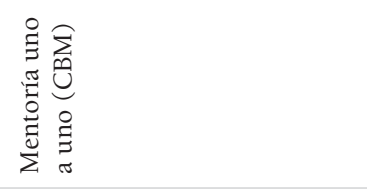 & & 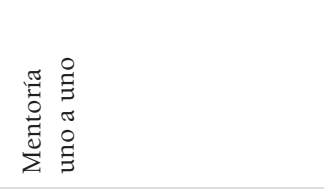 & 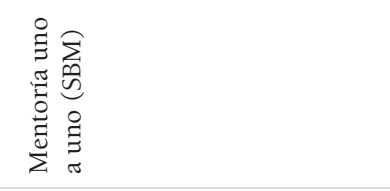 \\
\hline 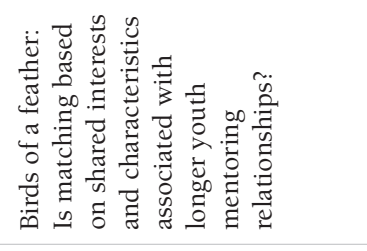 & & 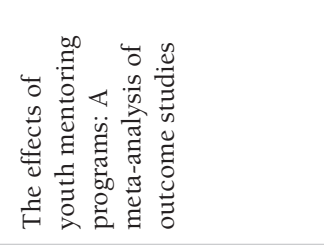 & 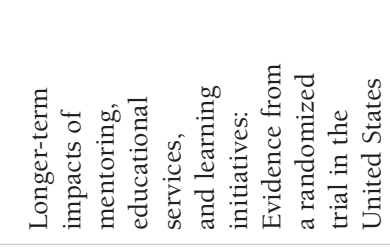 \\
\hline 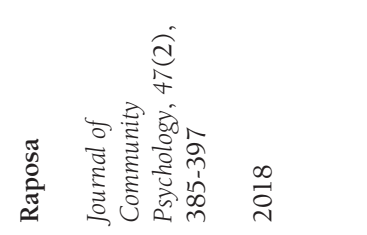 & & 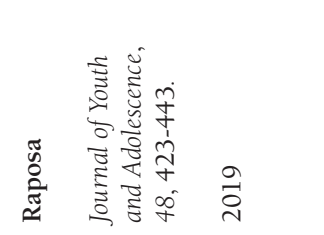 & 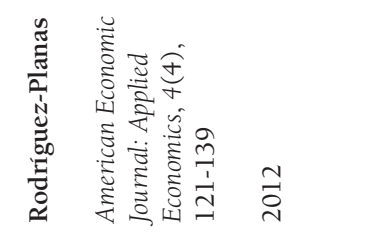 \\
\hline
\end{tabular}




\begin{tabular}{|c|c|c|}
\hline 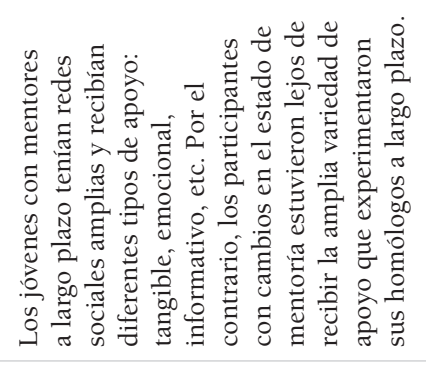 & 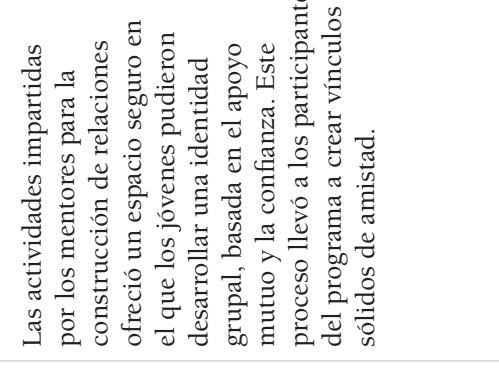 & 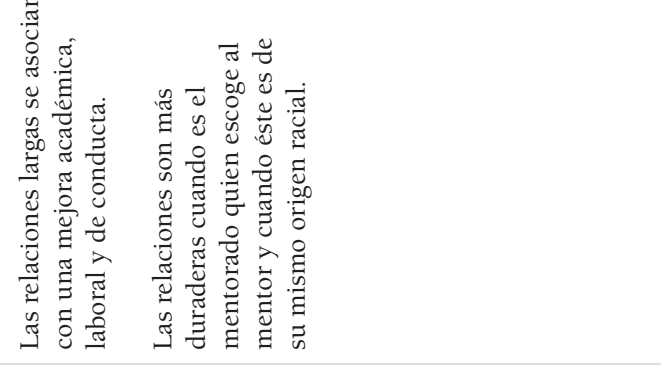 \\
\hline 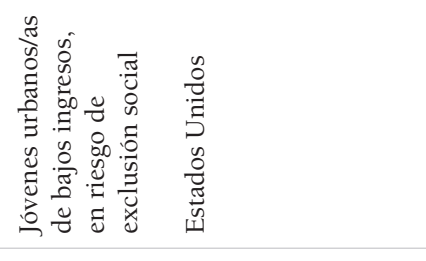 & 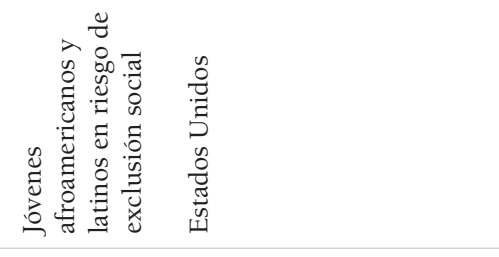 & 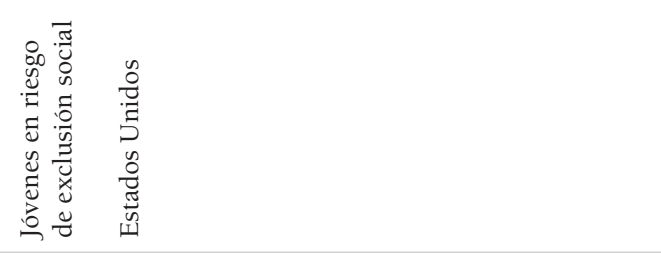 \\
\hline 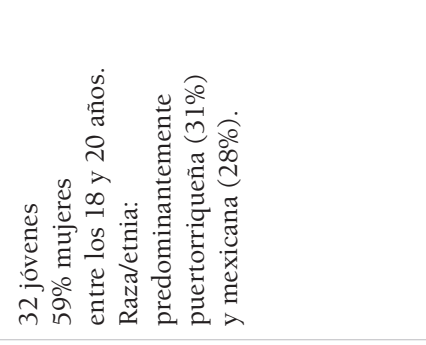 & 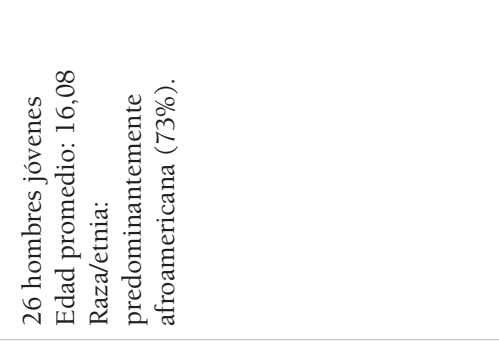 & 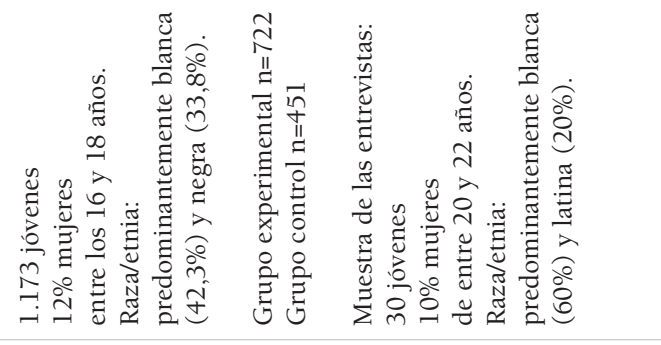 \\
\hline 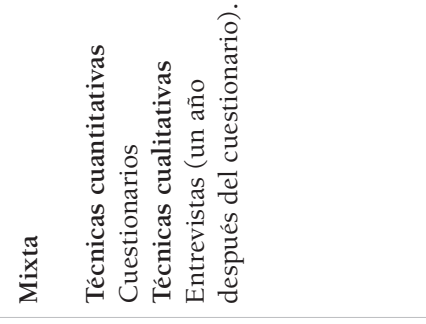 & 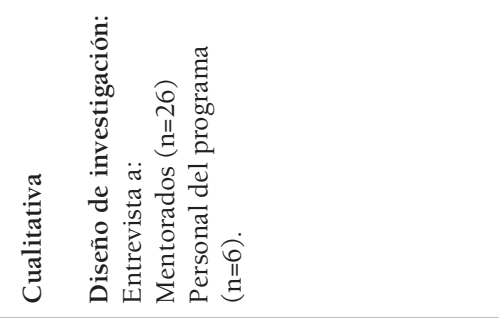 & 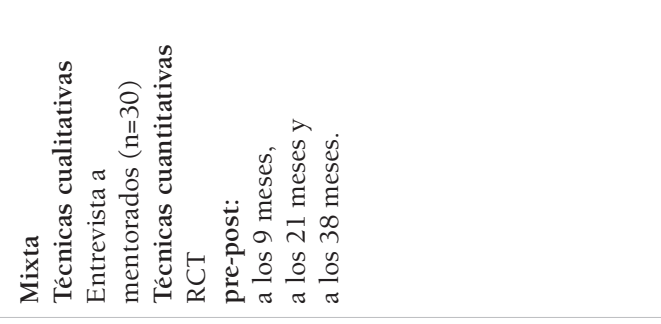 \\
\hline 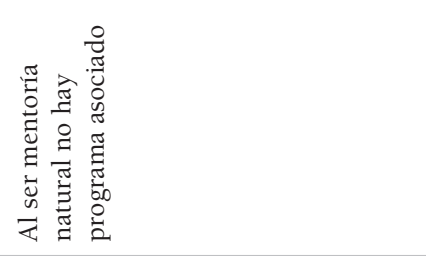 & 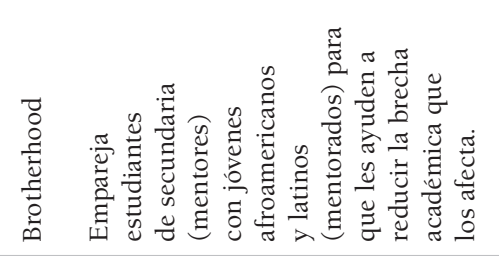 & 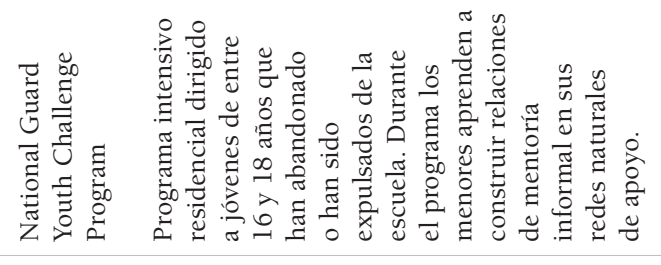 \\
\hline 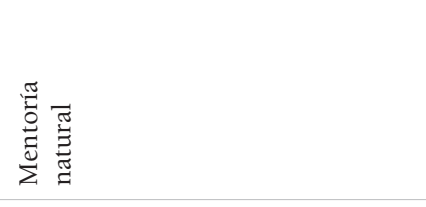 & 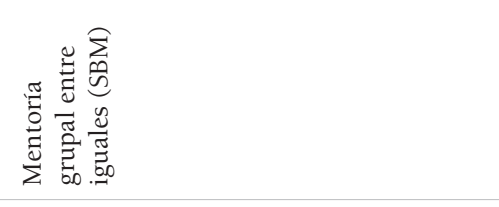 & 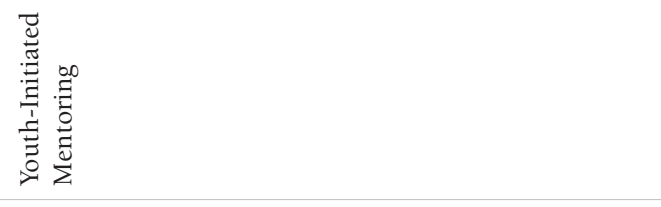 \\
\hline 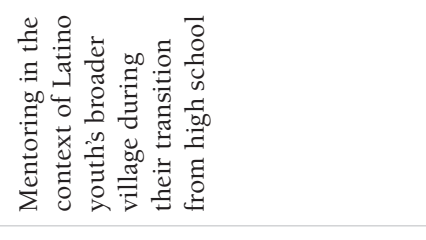 & 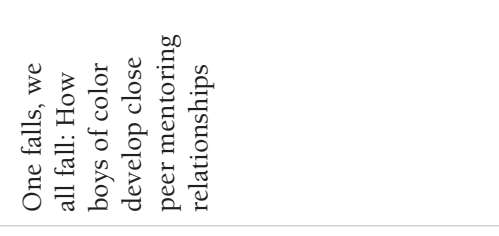 & 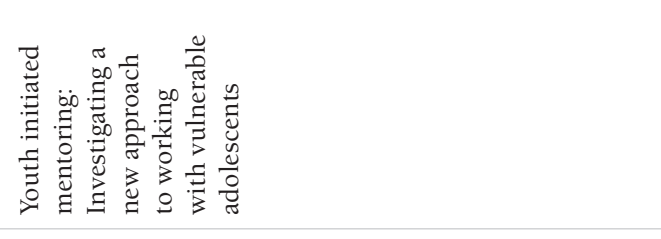 \\
\hline 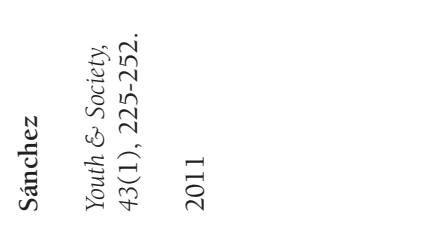 & 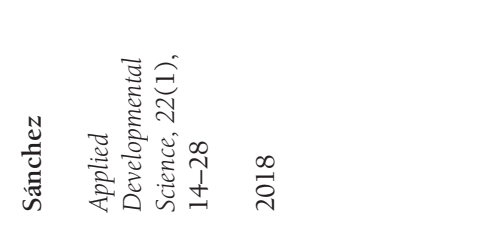 & 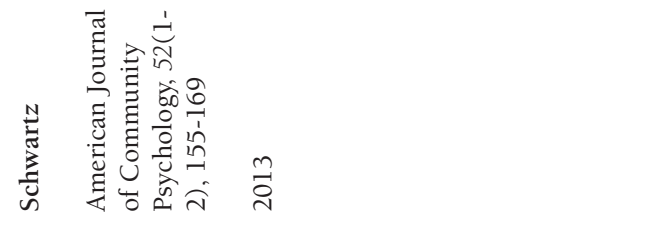 \\
\hline
\end{tabular}




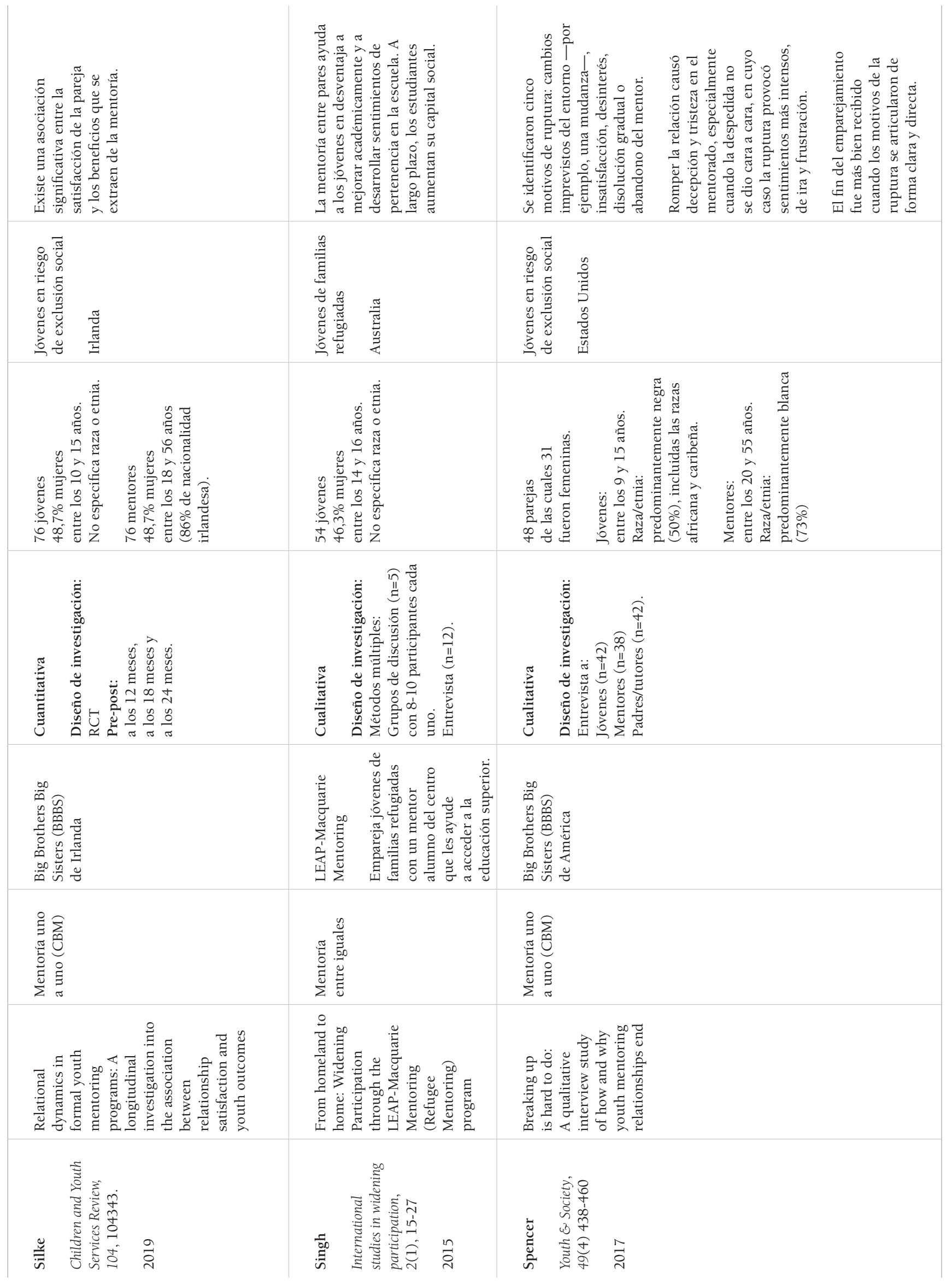




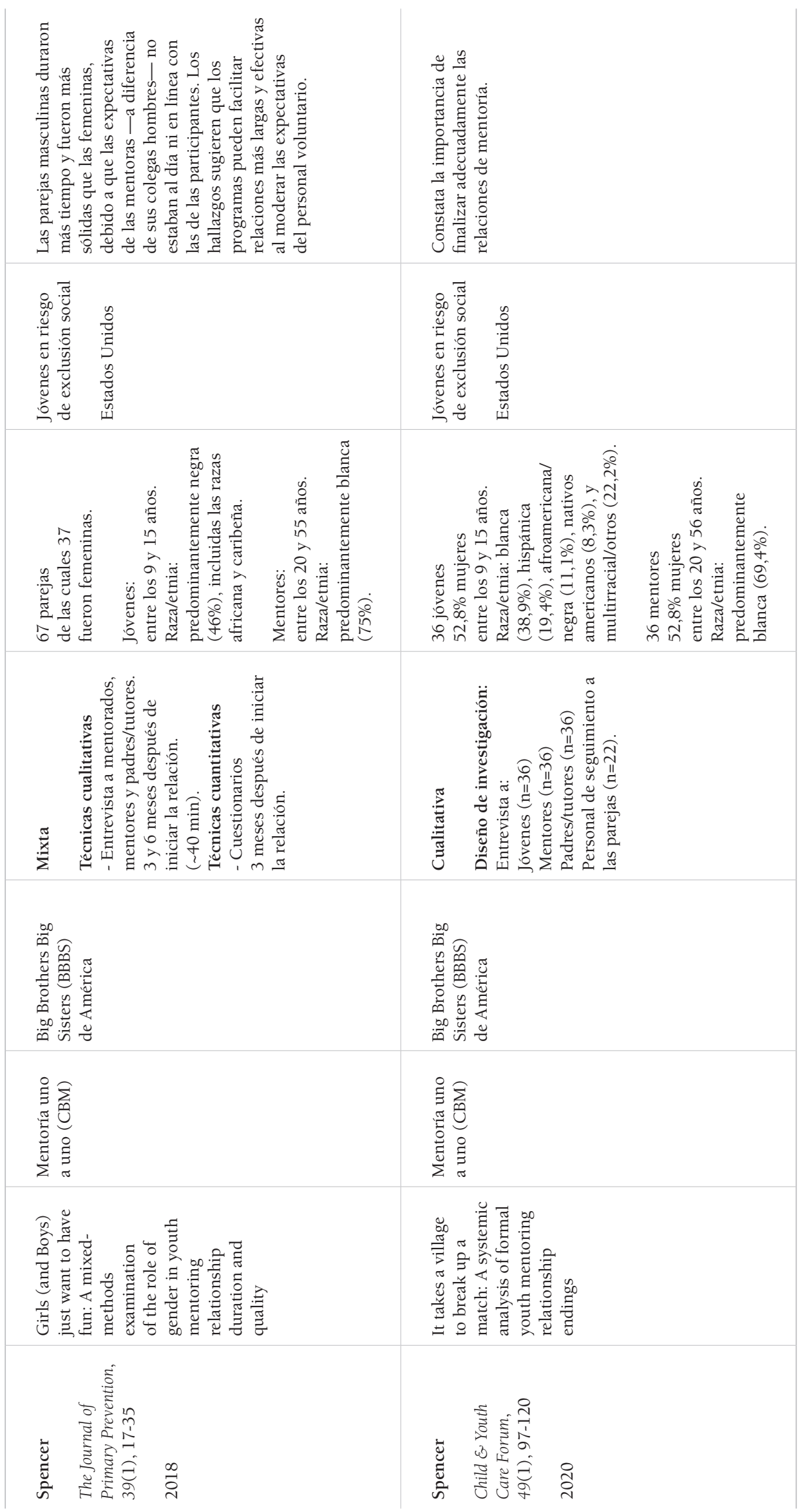




\begin{tabular}{|c|c|c|}
\hline 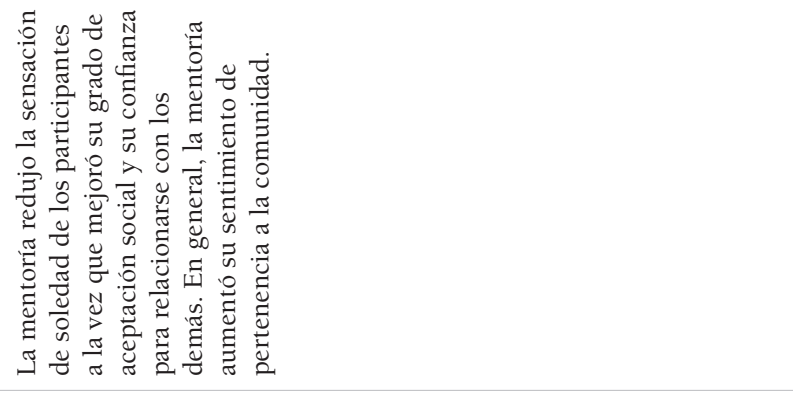 & 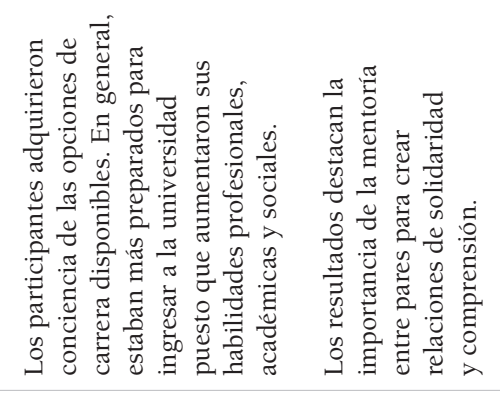 & 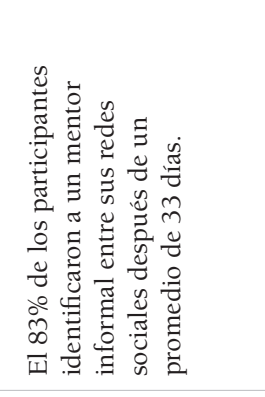 \\
\hline 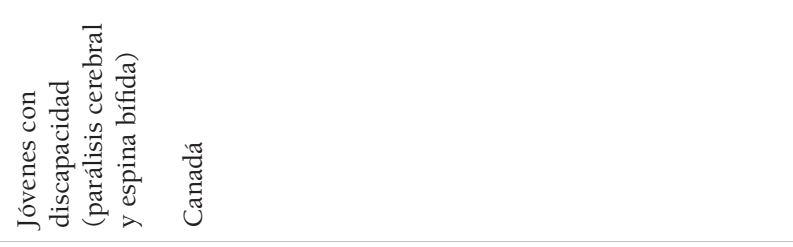 & 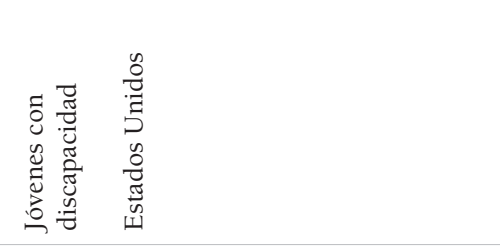 & 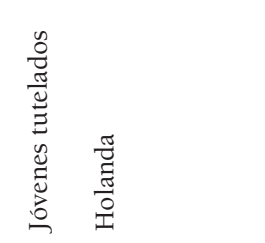 \\
\hline 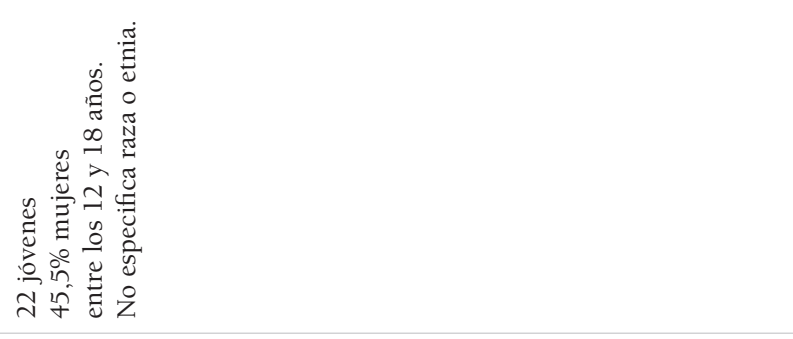 & 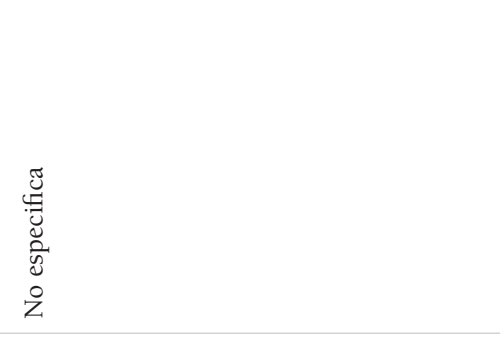 & 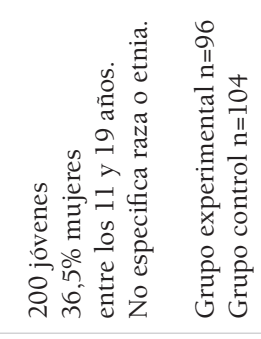 \\
\hline 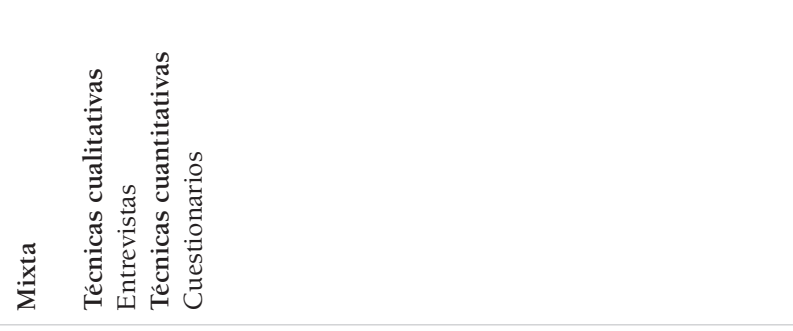 & 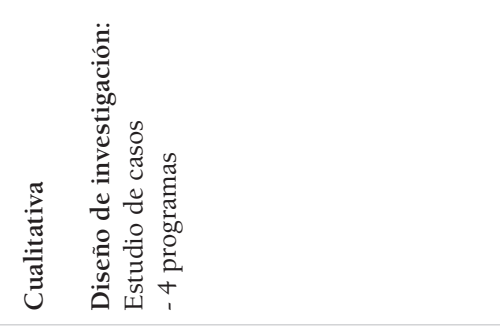 & 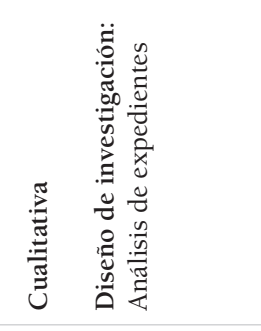 \\
\hline 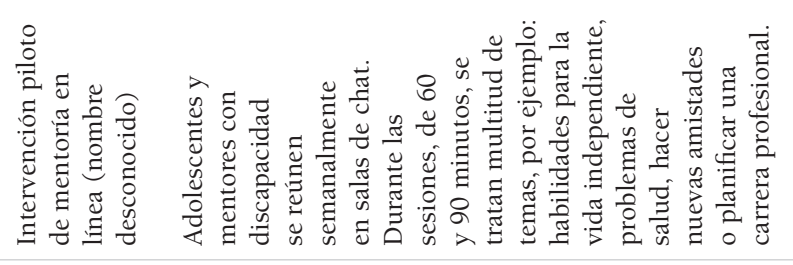 & 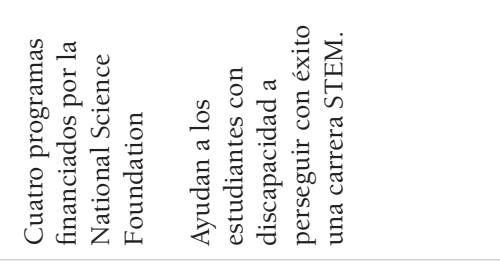 & 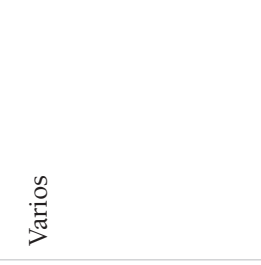 \\
\hline 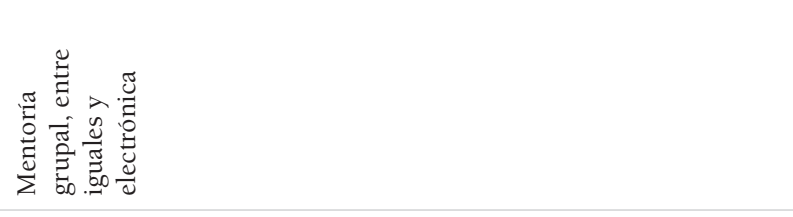 & 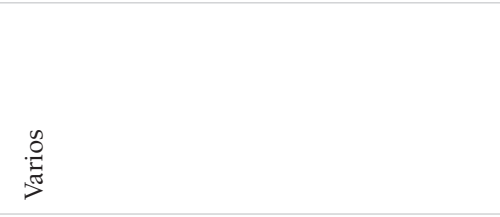 & 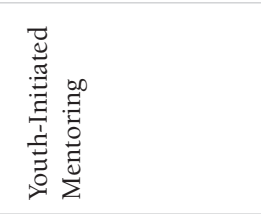 \\
\hline 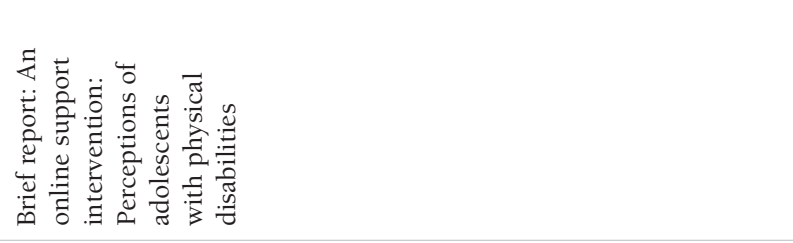 & 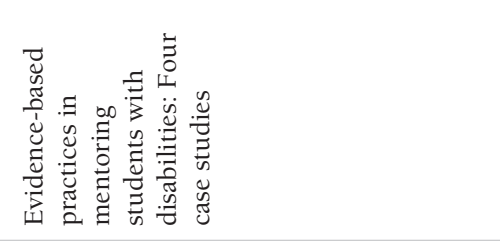 & 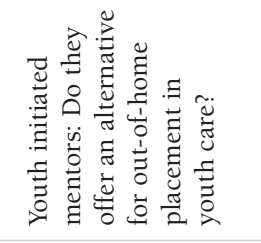 \\
\hline 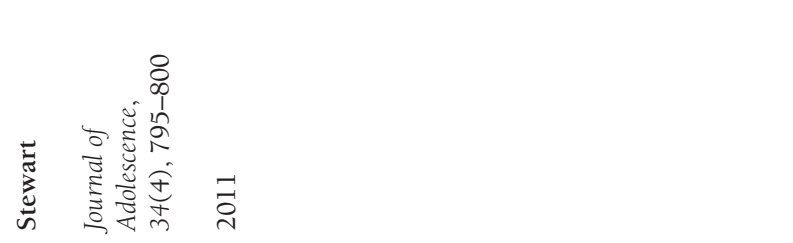 & 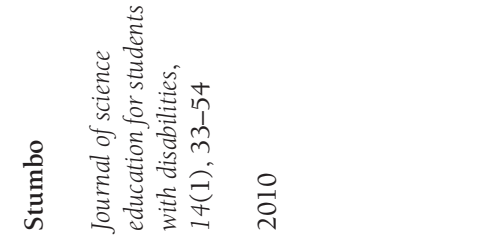 & 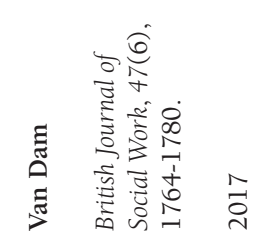 \\
\hline
\end{tabular}




\begin{tabular}{|c|c|c|}
\hline 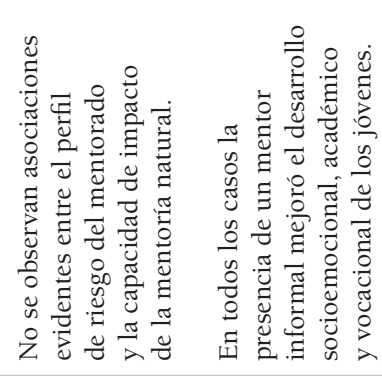 & 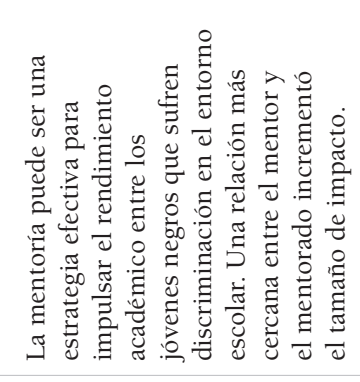 & 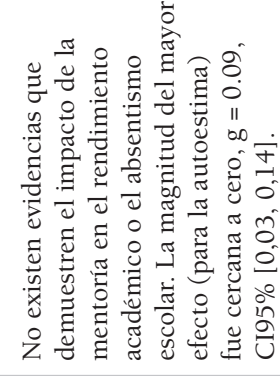 \\
\hline 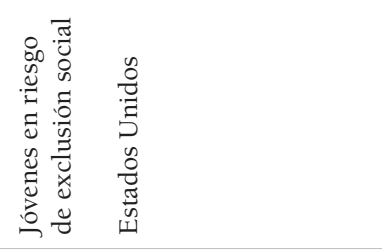 & 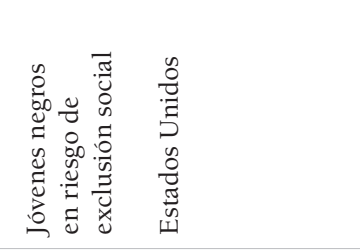 & 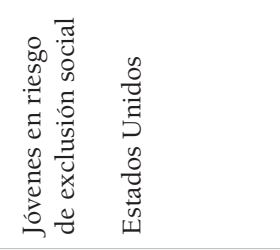 \\
\hline 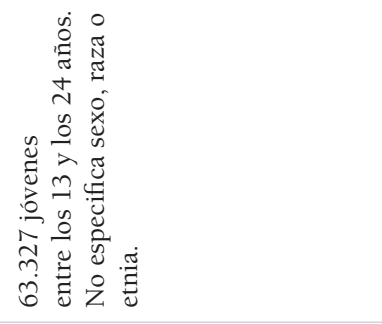 & 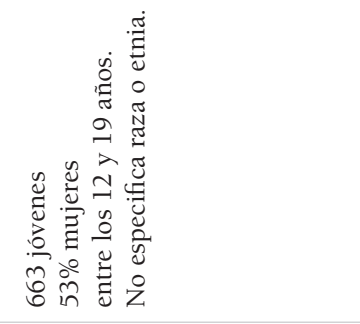 & 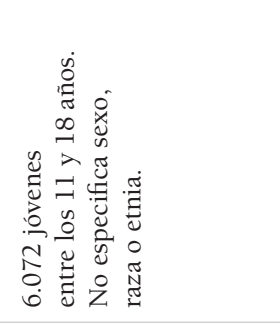 \\
\hline 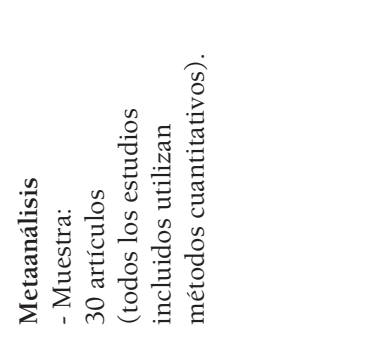 & 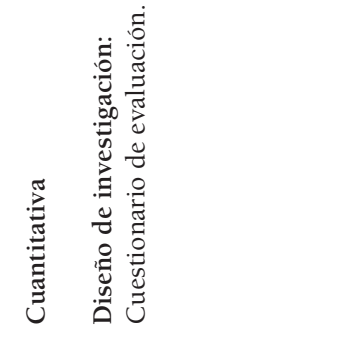 & 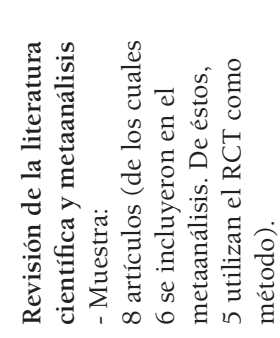 \\
\hline 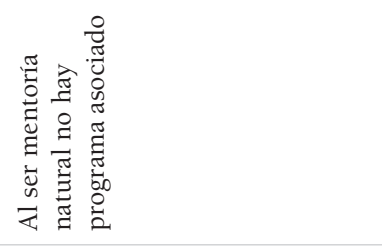 & 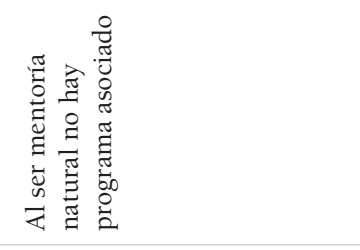 & 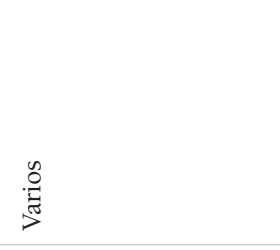 \\
\hline 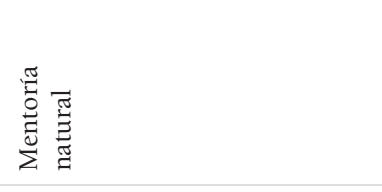 & 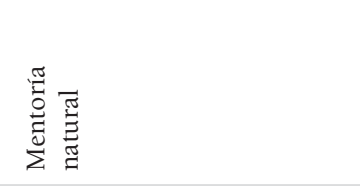 & 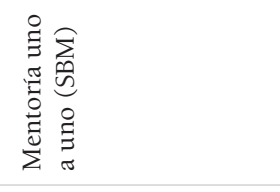 \\
\hline 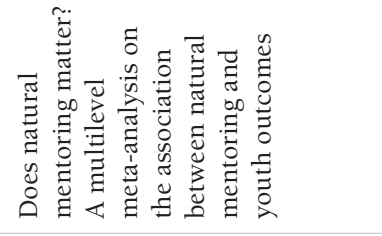 & 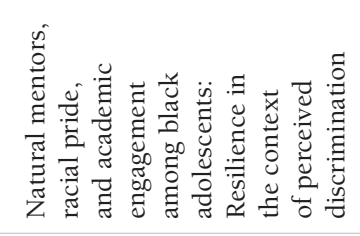 & 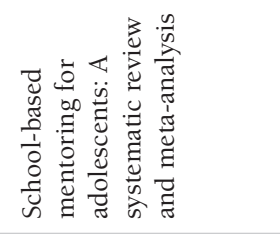 \\
\hline 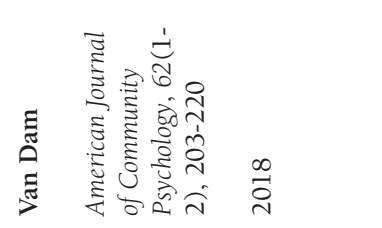 & 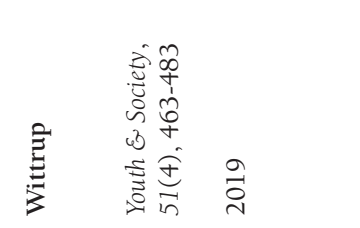 & 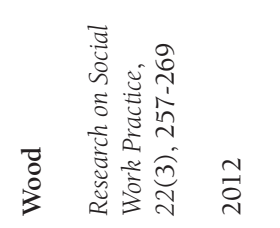 \\
\hline
\end{tabular}

